SUDAAR-762

\title{
PROTOCOL-BASED CONFLICT RESOLUTION FOR AIR TRAFFIC CONTROL
}

\author{
by
}

Inseok Hwang and Claire J. Tomlin

Department of Aeronautics And Astronautics

Stanford University, Stanford, CA 94305

JULY 2002 
SUDAAR-762

\title{
Protocol-based Conflict Resolution
}

\section{for Air Traffic Control *}

\author{
Inseok Hwang and Claire Tomlin \\ Hybrid Systems Laboratory \\ Department of Aeronautics and Astronautics \\ Stanford University, Stanford, CA 94305 \\ \{ishwang, tomlin@stanford.edu\}
}

\begin{abstract}
This paper proposes a method for multiple-aircraft conflict avoidance. We assume that aircraft cruise at constant altitude with varying velocities and that conflicts are resolved in the horizontal plane using heading change, velocity change, or a combination thereof. We assume that each aircraft's position, heading, and velocity are available to all aircraft involved in the conflict, we constrain the maneuver to be two straight paths of equal length, and we assume that all aircraft initiate conflict resolution maneuvers at the same time and that once an aircraft has initiated a maneuver, its velocity along the maneuver remains constant. Our multiple-aircraft conflict resolution methodology is presented in two steps; first, we consider an unrealistic but geometrically simple exact conflict, in which the original trajectories of all aircraft collide at a point, in order to derive a closed-form analytic solution for the required heading change, and then we consider a realistic inexact conflict, in which conflict points of multiple aircraft do not coincide. Heading change is a main control input for conflict resolution, yet velocity change is also used for an inexact conflict. We then construct a finite partition of the airspace around the conflict, and using our analytic solution, we derive a protocol for resolving the worst-case conflict within each partition. The result is a multiple-aircraft conflict resolution protocol, or a simple rule which is easily understandable and
\end{abstract}

* Research supported by NASA Ames under grant NASA/NCC 2-5422, by an NSF Career Award, and by the DARPA Software Enabled Control program, under grant F3361599-C-3014 (administered by AFRL). 
implementable by all aircraft involved in the conflict, and which provides guarantees of safety. We show that this solution is robust to uncertainties in the aircraft's position, heading, and velocity, as well as to path smoothing, and asynchronous maneuvers. We present simulation results for a dynamic aircraft model for various multiple aircraft conflict scenarios.

\section{Nomenclature}

We list nomenclature used throughout this paper; locally used nomenclature is explained in the corresponding section. We assume that the position coordinates are taken with respect to an origin which is at one of the original conflict points of the multiple aircraft conflict. In this paper, we use parameters from the data of B747 which cruises with Mach 0.8 at 40,000 ft altitude [5].

$\begin{array}{ll}r_{i} & \text { Radial position of aircraft } i, \text { nautical miles }(\mathrm{nm}) \\ \theta_{i} & \text { Angular position of aircraft } i, \text { radians }(\mathrm{rad}) \\ \left(x_{i}, y_{i}\right) & \text { Horizontal position of aircraft } i,(\mathrm{~nm}) \\ \psi_{i} & \text { Heading angle of aircraft } i,(\mathrm{rad}) \\ v_{i} & \text { Velocity of aircraft } i,(\mathrm{~nm} / \mathrm{sec}) \\ u & \text { Heading change for conflict resolution, }(\mathrm{rad}) \\ r_{\min } & \text { Minimum radius of the airspace partition, }(\mathrm{nm}) \\ r_{\max } & \text { Maximum radius of the airspace partition, }(\mathrm{nm}) \\ \diamond & \text { Destination point }\end{array}$

\section{Introduction}

This work is motivated by recent advances in navigation and data communication technologies which make it plausible for aircraft to fly their 
own trajectories instead of predefined paths in the National Airspace System [30, 14, 32, 22]. The idea of decentralizing some of the Air Traffic Control functionality to individual aircraft is part of the Free Flight concept, which has been studied by various research groups in the last several years $[26,29,31,36,37,38,12]$. In free flight, each aircraft could optimally choose its own trajectory to minimize fuel consumption and time delay. However, with the decentralization of control comes the requirement for providing clear and unambiguous methods for maintaining safe separation between aircraft. Thus, conflict detection and resolution (CD\&R) emerges as a critical issue for free flight.

Several recent papers have focused on solving CD\&R problems $[4,13$, $20,24,31,26,33,25,27,14,21,16,10,6,29,2,34,3,7,23,18,11,39,1$, $12,9,15,8,36,37]$. In a recent comprehensive survey, Kuchar and Yang [26] consider the problems of conflict detection and resolution separately; we focus on conflict resolution in this paper. The studies of conflict resolution may be categorized into three different cases according to the methods by which a solution is obtained. First, optimized conflict resolution produces a resolution maneuver which minimizes a given cost, a function of deviation from the original trajectory, flight time, fuel consumption, or energy. $[33,25,27,13,14,21,16,10,6,20,29,2,15,1]$. Menon et al.[29] consider conflict resolution as a multi-participant optimal control problem, which they solve using parameter optimization and state-constrained trajectory optimization. They compute a conflict resolution trajectory with two different costs: deviation from the original trajectory and a linear combination of total flight time and fuel consumption. Their method gives 3D optimal multiple-aircraft conflict resolution, yet the optimization process is in general computationally intensive. Bilimoria [4] proposes a method for pairwise geometric optimization which minimizes trajectory deviation; since it is a pairwise method, multiple-aircraft conflicts are resolved successively. In this method, conflict resolution maneuvers are obtained from a closedform analytic solution, and thus the method may be applied in real-time to two-aircraft conflicts; however, as we show in the next section, successive application of pairwise conflict resolution cannot guarantee safety for 
multiple-aircraft conflicts. Mao et al. [28] propose a decentralized conflict avoidance rule based on successive pairwise resolution and numerical optimization. However, this method cannot guarantee safety for multipleaircraft conflicts and is computationally intensive. Frazzoli et al. [13] use randomized searches to choose one from all possible cross patterns, and use convex optimization on the chosen cross pattern to obtain a resolution maneuver minimizing energy. Hu et al. [20] use braid theory to categorize types of multiple-aircraft resolutions, use a randomized algorithm to choose the type, and construct resolution maneuvers by solving a convex optimization problem to minimize the total length of trajectories (or energy) for a given type of maneuver. Due to the properties of convex optimization, the conflict resolution algorithms are numerically efficient, however since they are based on randomized algorithms, they could produce different solutions to the same conflict problems. Durand et al.[10] propose that predefined maneuvers be used to construct a multiple-aircraft conflict resolution maneuver; their method uses genetic algorithms to generate each resolution maneuver successively. Alliot et al. [1] solve multiple-aircraft conflicts by generating resolution maneuvers for each aircraft sequentially with a token allocation strategy; the $A^{*}$ algorithm is then used to select the optimal maneuver (minimum length trajectory) from a set of predefined maneuvers. Again, problems may occur when one attempts to apply successive pairwise resolution to multiple aircraft conflicts, as we show in the next section and as is indeed pointed at in Alliot et al.. In addition, the performance depends highly on the quality of the ordering: it is extremely difficult to find the optimal ordering $[10,1]$. Goodchild et al. [15] propose a cooperative optimal conflict resolution algorithm based on distributed artificial intelligence.

A second class of conflict resolution problems may be referred to as rule-based conflict resolution, in which a maneuver is resolved according to predescribed rules $[34,3,7,8]$. Duong et al.[8] propose Extended Flight Rules, which extends the current Visual Flight Rules by assigning a priority to each aircraft involved in a conflict. The rules may be simple to understand and easily implementable, but they do not properly account for unexpected events. For example, a conflict situation may fall into several predefined 
cases due to uncertainties in the aircraft's position and heading; in these cases, the ambiguity in which rule to choose may lead to an unsafe resolution. The method may also require many rules to completely cover all possible conflicts.

A third class of conflict resolution techniques uses force field methods and assumes aircraft fly in the force field generated by a potential function; the forces induced by the potential function form a resolution maneuver[23, 18, 11, 39, 9, 40]. Duong and Zeghal [9] present a technique in which a force field "generated" by an intruding aircraft produces a conflict avoidance action and a force from the flight plan generates an attracting action. Zeghal and Ferber [40] propose a distributed conflict resolution algorithm based on a symmetrical force field method. They give a resolution maneuver using a relatively simple equation, yet the resolution maneuver may have several discontinuities which aircraft cannot follow; most importantly, safety cannot be proven about such multiple aircraft maneuvers.

This paper presents a protocol-based conflict resolution method for multiple aircraft in air traffic control. Aircraft are assumed to cruise at the same altitude with varying velocities. Each aircraft's position, velocity, and heading are assumed to be available to all aircraft which are involved in the conflict; this assumption can be justified due to the proposed availability of the Global Positioning System (GPS) [14] and a new datalink between aircraft known as Automatic Dependent Surveillance-Broadcast (ADS-B) [35]. We assume synchronous maneuvers, in which all aircraft change their heading for conflict resolution at the same time. Even though the synchronous maneuver assumption is common to most recent multiple-aircraft conflict resolution methods, we show in Section 7.3 that our protocol is robust with respect to asynchronous maneuvers, in which all aircraft may not necessarily change their heading at the same time. A conflict resolution maneuver is composed of two straight paths which have the same length. During the resolution maneuver, each aircraft involved in the conflict resolution is assumed to keep its velocity constant. We derive the maneuver first for the exact conflict case, which represents the (unrealistic) situation in which all aircraft collide at a point in time and space; we then use these results to 
generalize to the inexact conflict case, in which conflict points of multiple aircraft do not coincide in time and space. Heading change from the original path is the primary control input and is used exclusively when the conflict is exact, or close to exact, meaning that the points of all aircraft conflicts are close to each other; velocity change is also used as a control input when an exact conflict assumption fails. Kinematic aircraft models are used to derive the protocol. Our use of heading change (instead of altitude change) is motivated by current airspace structure, in which aircraft fly in a vertically stratified airspace.

We derive for the exact case a closed form analytic solution for the required heading change of all aircraft to resolve the conflict. For the inexact case, we derive an analytic solution to a conflict resolution according to the conflict configuration. We then construct a finite partition of the airspace around the conflict, and using our analytic solution, we derive a protocol for resolving the worst-case conflict within each partition. The result is a simple rule, easily understandable and implementable by all aircraft involved in the conflict, and well defined for a wide range of initial aircraft configurations. Since the protocol is obtained from a closed-form analytic solution, it can be implemented in airborne systems for real-time conflict resolution, as well as in ATC ground systems. If an aircraft lies within the airspace partition around a conflict point, the aircraft must follow the protocol for safety even if it is not involved in the conflict; because we treat the multiple-aircraft case directly and all aircraft within the airspace partition take the resolution maneuvers, we avoid potential problems and the "domino" effect which arises when pairwise collision avoidance is performed sequentially. Most importantly, we provide guarantees of safety of this protocol. This is the main difference between the method proposed in this paper and many other currently available multiple aircraft $C D \& R$ solutions. Our methods may not be optimal in the sense that some conflicts can be resolved with smaller deviations from the desired trajectory than our protocol produces. However, our protocol is implementable in real time, and always guarantees safe conflict resolution. In addition, we can reduce any conservatism in our protocol by using a finer partition. We show that the solution is robust to uncertain- 
ties in the aircraft's position, velocity, and heading, and that the method remains safe when sharp corners on the resolution trajectories are replaced by circular arcs. Though a kinematic model is used to design the protocol, a dynamic model is used for validation and to simulate real situations.

This paper is organized as follows: our motivation for multiple aircraft conflict resolution is presented in Section 2. Section 3 presents the model and problem formulation. The safety conditions for conflict resolution and the protocol for multiple-aircraft conflict resolution for an exact conflict are derived in Section 4 and Section 5 respectively. The protocol for multipleaircraft conflict resolution for an inexact conflict is derived in Section 6 . In Section 7, we analyze the robustness of the protocols with respect to uncertainties in parameters such as position, velocity, and heading, as well as to smoothing corners by circular arcs, and to asynchronous maneuvers. Conclusions are presented in Section 8. Appendix A gives a proof of the safety condition for multiple-aircraft conflict resolution for an exact conflict, and the validation of the protocols for multiple-aircraft conflict resolution with a dynamic aircraft model and random simulations are presented in Appendix B.

\section{Motivation for multiple-aircraft conflict resolu- tion}

Multiple-aircraft conflict resolution is motivated by the fact that pairwise conflict resolution is not guaranteed to resolve multiple-aircraft conflicts. In this section, we illustrate by means of an example that optimal pairwise resolution, when applied successively to multiple aircraft problems, may result in unsafe or ambiguous situations.

For example, consider the three-aircraft conflict situation illustrated in Figure 1-(a). We apply an optimal geometric pairwise conflict resolution [4] to this scenario. This conflict resolution is assumed to be noncooperative: only one aircraft involved in the conflict takes a resolution maneuver and the other follows its original trajectory. An optimal solution is one which minimizes the amount of deviation from the nominal trajectory for the deviating 


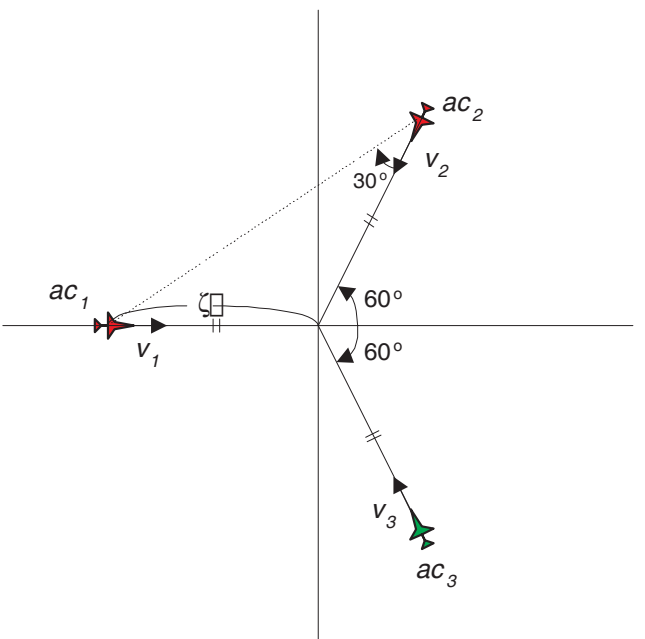

(a) Initial conflict configuration.

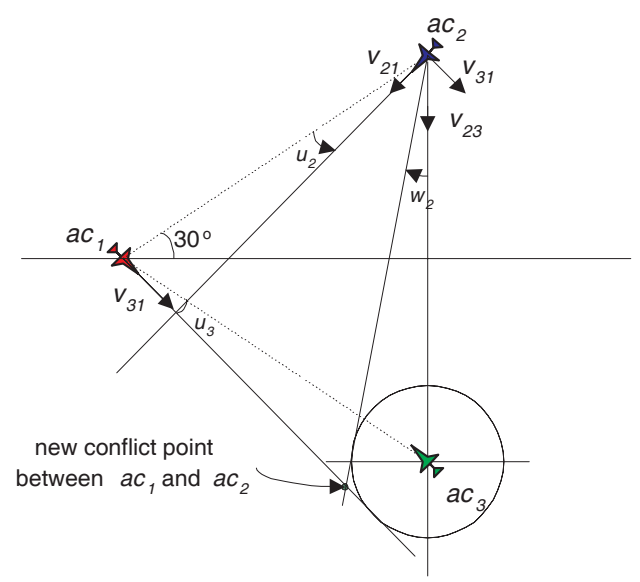

(c) Conflict resolution in the relative coordinate attached to $\mathrm{ac}_{3}$.

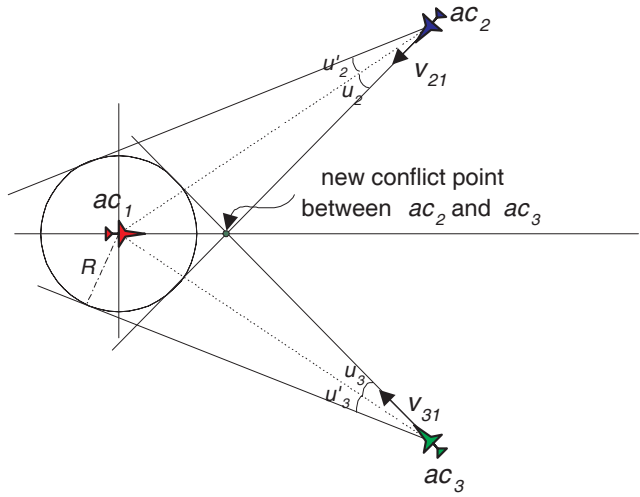

(b) Conflict resolution in the relative coordinate attached to $\mathrm{ac}_{1}$.

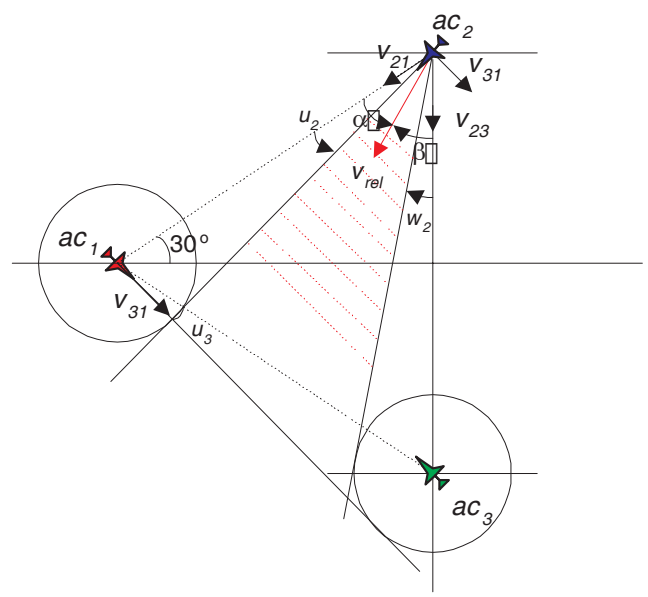

(d) Safe conflict resolution solution.

Figure 1: Three-aircraft conflict resolution with optimal geometric pairwise resolution; illustrating how successive application of pairwise collision avoidance results in unsafe situation. 
aircraft. If the velocity vector is held constant after the resolution maneuver commences, the minimum trajectory deviation is the one that minimizes the magnitude of the velocity vector change. A conflict resolution maneuver can be obtained by using heading change only, velocity change only, or heading-velocity change.

A time horizon of $\left[0, T_{f}\right]$ is assumed, where 0 is an initial time at which all aircraft involved in a conflict initiate their conflict resolution maneuver and $T_{f}$ is a maneuver completion time for all aircraft. A conflict is defined to be the event in which the distance between any pair of aircraft, $d(t)$, is less than that of predefined safety distance, $R$, at some $t \in\left[0, T_{f}\right] \Leftrightarrow$ $\exists t \in\left[0, T_{f}\right]$ such that $d(t)<R(R$ is assumed to be 5 nautical miles (nm) in this paper). Then, safety means there are no conflicts over $\left[0, T_{f}\right]$, i.e. the minimum distance between any pair of aircraft is greater than or equal to $R$. Each aircraft's velocity is bounded by a minimum allowable velocity, $v_{\min }$, and a maximum allowable velocity, $v_{\max }: v \in\left[v_{\min }, v_{\max }\right]$. First, we consider heading change only as a control input for conflict resolution. We assume all three aircraft velocities are the same and constant, and conflict detection and heading change are assumed to be instantaneous. Then, an optimal resolution which minimizes trajectory deviation is the one which minimizes heading change. We consider the conflict situation in the relative coordinate attached to aircraft 1 as shown in Figure 1-(b). Conflict detection and resolution take place when aircraft reaches a point whose distance from a conflict point is $\zeta$. Aircraft 2 and aircraft 3 detect separate conflicts with aircraft 1 , simultaneously, and must change their headings at least by $u_{2}$ (or $u_{2}^{\prime}$, due to turn ambiguity) and $u_{3}$ (or $u_{3}^{\prime}$ ) respectively to avoid a conflict. Without loss of generality, we assume aircraft 2 and aircraft 3 change their heading by $u_{2}$ and $u_{3}$ respectively. Immediately after changing their heading, aircraft 2 and aircraft 3 detect a new conflict between themselves as shown in Figure 1-(b), or in relative coordinates attached to aircraft 3 in Figure 1 -(c). Since the relative velocity of aircraft $2, v_{23}$, goes through aircraft 3 , it is clear that there is a conflict between aircraft 2 and aircraft 3. Aircraft 2 changes its heading by $w_{2}$ in order to resolve the conflict with aircraft 3 , yet there is a new conflict between aircraft 1 and aircraft 2 as shown 
in Figure 1-(c). This phenomenon is called a domino effect. In order to resolve the conflicts, the relative velocity of aircraft $2, v_{r e l}$ must eventually lie within the shaded cone as shown in Figure 1-(d): if we define $\alpha$ as an angle between $v_{r e l}$ and $v_{21}$ in the relative coordinate attached to aircraft 1 , then $u_{2}-\alpha \leq 0$ for safety, and similarly, if $\beta$ is defined as an angle between $v_{r e l}$ and $v_{23}$ in the relative coordinate attached to aircraft $3, w_{2}-\beta \leq 0$ for safety. Consider the following numerical examples, in which $\zeta$ is chosen as $60 \mathrm{~nm}$ [4], 50nm, 40nm, 30nm, and $15 \mathrm{~nm}$. We assume all three aircraft fly with $v=7.5 \mathrm{~nm} / \mathrm{min}$ (which comes from the data of a B747 cruising with $M=0.8)$.

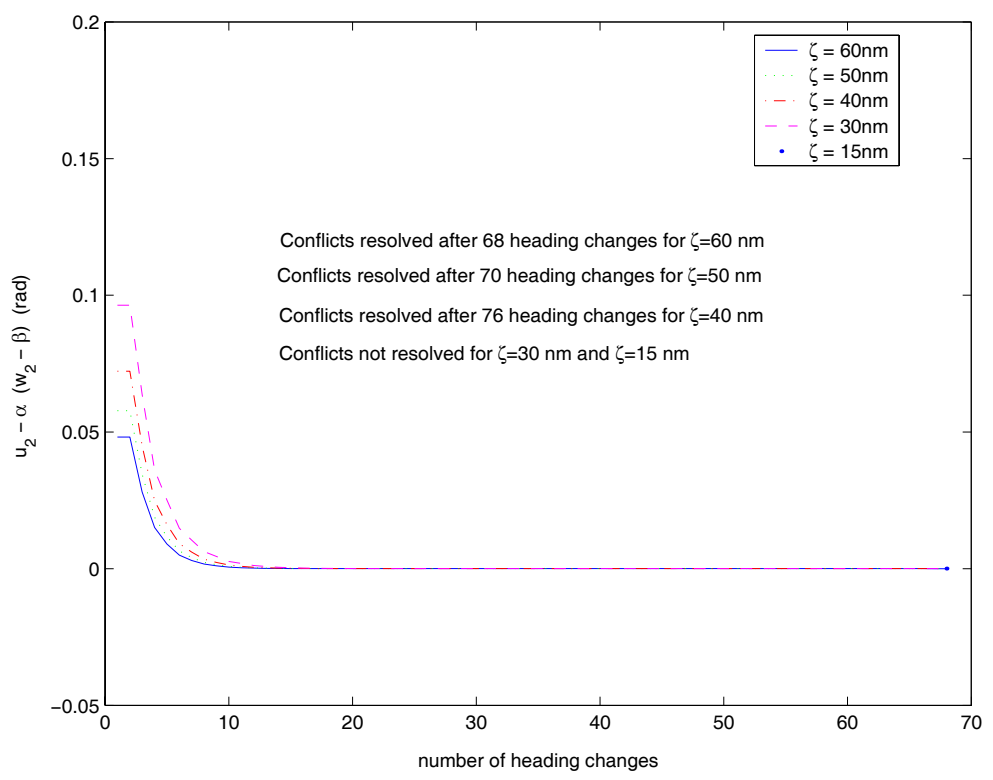

Figure 2: Heading difference of aircraft 2 between $v_{r e l}$ and $u_{2}\left(\right.$ or $\left.w_{2}\right)$ in unsafe situation.

As shown in Figure 2, for $\zeta=60 \mathrm{~nm}$, resolution occurs after 68 iterations of the above pairwise algorithm, which may not be practical in real time applications. As $\zeta$ decreases, the number of heading changes required increases until finally the three-aircraft conflict cannot be resolved safely when $\zeta=30 \mathrm{~nm}$ since an infinite number of heading changes takes place in finite time. Even though the unsafe conflict in the above example can be 
avoided by choosing the opposite direction of heading change, the heading turn ambiguity itself may cause an unsafe conflict resolution.

If we assume speed change to be a control input for conflict resolution instead of heading change, then a large class of conflicts (even two-aircraft) cannot be resolved.

Finally, unsafe situations may arise when the resolutions are optimal heading-velocity change, in which heading and velocity are changed simultaneously. If the resulting optimal velocity $\left(v^{o p t}\right)$ is not in $\left[v_{\min }, v_{\max }\right]$, a new resulting optimal speed is $v_{\text {new }}^{o p t}=v_{\min }$ if $v^{o p t}<v_{\text {min }}$ and $v_{\text {new }}^{o p t}=v_{\text {max }}$ if $v^{\text {opt }}>v_{\max }$. With this fixed optimal velocity, we may repeat the analysis of heading change above to show that the domino effect, and unsafe situations, may still arise.

Several recent papers have focused on the multiple-aircraft conflict resolution case. However, their approaches are based on optimization procedures and randomized algorithms[13, 20, 29], and thus could produce different solutions to identical problems, which may cause confusion in real applications. Since optimization procedures are computationally intensive, they are undesirable for real-time airborne applications.

Thus, in this paper we propose a protocol-based conflict resolution for multiple-aircraft conflicts to overcome the above problems. Our algorithm has the following properties. Since the multiple-aircraft case is treated directly, potential problems and the domino effect shown in the above example can be avoided. Our algorithm is based on an analytic solution for the control input and we implement it via a partition around the conflict. Our solution is simple to understand, deterministic, and provably safe. Since it is based on a closed-form analytic solution, it can be implementable in airborne systems for real-time conflict resolution. Our methods are not optimal, in the sense that some conflicts can be resolved with smaller deviations from the desired trajectory than our protocol produces, yet the conservatism of our protocol is reduced as we form a finer partition of the airspace. However, since our method is based on the assumption that all aircraft involved in a conflict have information about all other aircraft, it may not be robust to information loss and partial information. 
Finally, we remark that while the majority of conflicts occurring in the current airspace are two-aircraft conflicts, multiple aircraft methods are important for two reasons. First, a method that evolves gracefully from treatment of two aircraft to treatment of multiple aircraft conflicts would be efficient in resolving even today's small number of multiple aircraft problems; second, as the airspace and air traffic system evolve to a stage in which aircraft are more often flying user preferred routes, one would expect more multiple aircraft conflicts.

\section{Model and problem formulation}

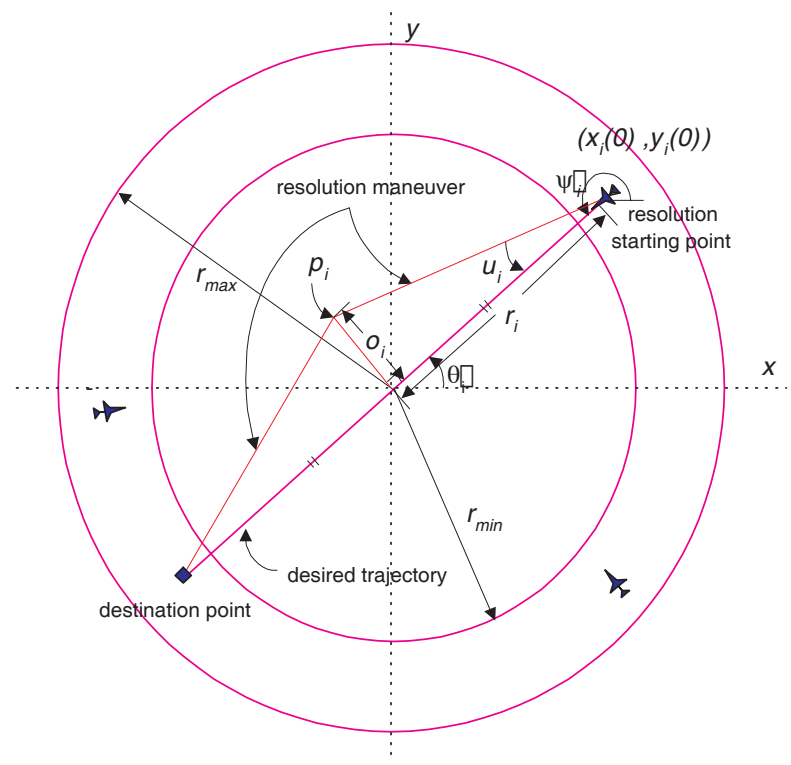

Figure 3: An illustration of the exact conflict resolution maneuver for one of $N$-aircraft and parameters involved.

We consider the problem of conflict resolution in the horizontal plane, using only information about each aircraft's current position, velocity, and heading. As in the previous section, we define a conflict to be the event in which, at any time during a given time horizon, the distance between any 
pair of aircraft is less than $R$, which we assume to be $5 \mathrm{~nm}$. We consider the following two cases:

Definition 1 (Exact conflict) All aircraft involved in a conflict come into conflict at a single point in space and time.

Definition 2 (Inexact conflict) At least one of the aircraft involved in a conflict comes into conflict at a different point in space and time than the others.

In this section, we present the model and formulate the collision avoidance problem for the exact conflict case; the more general inexact conflict case will be detailed in Section 6, using analysis and results from this and following sections.

We assume that each aircraft has a desired, or nominal, trajectory, which is a straight path of constant heading. Conflict detection occurs by simple projection of this nominal trajectory ahead by a fixed time, which we assume in this paper to be greater than 20 minutes, though our method is general enough to work for any finite time horizon. When a conflict is detected, all aircraft involved in the conflict prepare to initiate a conflict resolution maneuver. The maneuver is assumed to start at (conflict-time-T), where conflict-time is the time at which the aircraft would have reached the conflict point, and $T$ is a fixed time, assumed in this paper to be 20 minutes. In our notation, we thus initiate the time horizon $\left[0, T_{f}\right]$ so that (conflict-time$T)=0$. The aircraft involved in the conflict are assumed to be flying at constant velocity during a resolution maneuver. The velocities are bounded between known values $v_{\min }$ and $v_{\max }$.

We initially partition the airspace around the conflict point into two concentric circular discs of radii $r_{\min }$ and $r_{\max }$, as shown in Figure 3 . These radii are designed so that $r_{\min }=v_{\min } T$ and $r_{\max }=v_{\max } T$, which ensures that the aircraft lie in the annulus between the two radii at the initiation of the conflict resolution maneuver.

For aircraft $i$, we denote the starting position as $\left(x_{i}(0), y_{i}(0)\right)$ such that

$$
\left[\begin{array}{c}
x_{i}(0) \\
y_{i}(0)
\end{array}\right]=\left[\begin{array}{c}
r_{i} \cos \theta_{i} \\
r_{i} \sin \theta_{i}
\end{array}\right], \quad \text { where } i=1,2, \cdots, N
$$


where $r_{i}=v_{i} T$ as shown in Figure 3 .

For aircraft $i$, the destination point is computed as the point at which the aircraft must "rejoin" its original trajectory after completing the conflict resolution maneuver. This point is assumed to be an equal distance along the desired trajectory from the conflict point, as the conflict point is from $\left(x_{i}(0), y_{i}(0)\right)$. We assume that the aircraft can change heading instantaneously, and that the control input is a piecewise constant heading change $\left(\Delta \psi_{i}\right)$ which we denote as $u_{i}$. While we assume an instantaneous heading change, we will show later in this paper that the protocol is still valid when a sharp corner in the resulting $\left(x_{i}, y_{i}\right)$ path is replaced with a smooth circular arc. Also, we assume synchronous maneuvers, in which all aircraft change their heading at the same time, yet we will show that our protocol is robust with respect to asynchronous maneuvers, in which all aircraft may not necessarily change their heading at the same time. We assume $u_{i}$ to be the same for all aircraft. The resolution maneuver for each aircraft is set to be an isosceles triangular path composed of two straight segments of constant heading and of constant velocity, the apex of which is the new waypoint, $p_{i}$, connecting the initial position $\left(x_{i}(0), y_{i}(0)\right)$ with the destination point, as shown in Figure 3. The position of the waypoint $p_{i}$, and the vertical deviation $o_{i}$, is determined up to sign by the heading change $u_{i}$. It can easily be shown that under this maneuver, all aircraft arrive at their waypoints $p_{i}$, and their destination points, at the same time. Thus the problem of maneuver protocol design reduces to computing the heading change $u_{i}$ to ensure safety.

We use a kinematic model for each aircraft to design the protocol:

$$
\left[\begin{array}{c}
\dot{x}_{i} \\
\dot{y}_{i}
\end{array}\right]=\left[\begin{array}{c}
v_{i} \cos \psi_{i} \\
v_{i} \sin \psi_{i}
\end{array}\right], \quad \text { where } i=1,2, \cdots, N
$$

Finally, in this design, we are assuming that all aircraft share information about the other aircraft's position, velocity, and heading. This information sharing can be achieved by communication through Automatic Dependent Surveillance-Broadcast (ADS-B). In our current work, we are analyzing the effect on the protocol of differing information horizons. 


\section{Derivation of safety conditions for multiple-aircraft conflict resolution for an exact conflict}

In this section, we derive the safety conditions for multi-aircraft conflict resolution for an exact conflict. First, a necessary and sufficient condition for safety of multiple-aircraft conflict resolution is derived. Since the safety condition for the proposed conflict resolution is based on the minimum distance between aircraft, the geometry of the conflict resolution maneuver is symmetric, and all aircraft change their heading at the same time, the safety condition can be derived over the first half and the second half of the conflict resolution path separately. Here, the safety condition is based on the assumption of an exact collision; we show in Section 6 that the same safety condition is also used for an inexact conflict resolution.

To ensure there is no conflict during the resolution maneuver, the minimum distance between aircraft should be greater than or equal to the predefined safety distance, $R$. For notational simplicity, we define for aircraft $i$ and $j: \phi_{i}:=\psi_{i}+u_{i}, a:=v_{i} \cos \phi_{i}-v_{j} \cos \phi_{j}, \quad b:=r_{i} \cos \theta_{i}-r_{j} \cos \theta_{j}$, $c:=v_{i} \sin \phi_{i}-v_{j} \sin \phi_{j}, \quad e:=r_{i} \sin \theta_{i}-r_{j} \sin \theta_{j}$.

Then, the distance squared between aircraft $i$ and aircraft $j$ on the first half path is:

$$
\begin{aligned}
S_{i j}^{1}(t)= & \left(x_{i}(t)-x_{j}(t)\right)^{2}+\left(y_{i}(t)-y_{j}(t)\right)^{2} \\
= & {\left[\left(v_{i} \cos \phi_{i}-v_{j} \cos \phi_{j}\right) t+\left(r_{i} \cos \theta_{i}-r_{j} \cos \theta_{j}\right)\right]^{2} } \\
& +\left[\left(v_{i} \sin \phi_{i}-v_{j} \sin \phi_{j}\right) t+\left(r_{i} \sin \theta_{i}-r_{j} \sin \theta_{j}\right)\right]^{2} \\
= & (a t+b)^{2}+(c t+e)^{2}
\end{aligned}
$$

where $0 \leq t \leq \frac{T_{f}}{2}$, and $i, j=1,2, \cdots, N, i \neq j$.

Similarly, we define: $\chi_{i}:=\psi_{i}-u_{i}, \lambda:=v_{i} \cos \chi_{i}-v_{j} \cos \chi_{j}, \quad \mu:=$ $r_{i} \sin \chi_{i}-r_{j} \sin \chi_{j}, \xi:=\frac{1}{2} T_{f} a+b, \quad \sigma:=\frac{1}{2} T_{f} c+e$.

Then, the distance squared between aircraft $i$ and aircraft $j$ on the second 
half path is:

$$
\begin{aligned}
S_{i j}^{2}(\tau)= & {\left[\left(v_{i} \cos \chi_{i}-v_{j} \cos \chi_{j}\right) \tau+\left(v_{i} \cos \phi_{i}-v_{j} \cos \phi_{j}\right) \frac{T_{f}}{2}\right.} \\
& \left.+\left(r_{i} \cos \theta_{i}-r_{j} \cos \theta_{j}\right)\right]^{2}+\left[\left(v_{i} \sin \chi_{i}-v_{j} \sin \chi_{j}\right) \tau\right. \\
& \left.+\left(v_{i} \sin \phi_{i}-v_{j} \sin \phi_{j}\right) \frac{T_{f}}{2}+\left(r_{i} \sin \theta_{i}-r_{j} \sin \theta_{j}\right)\right]^{2} \\
= & (\lambda \tau+\xi)^{2}+(\mu \tau+\sigma)^{2}
\end{aligned}
$$

where $0<\tau \leq \frac{T_{f}}{2}$, and $i, j=1,2, \cdots, N, i \neq j$.

In our problem setting, the worst case occurs when the minimum distance among aircraft is achieved. If the minimum distance between aircraft is always greater than or equal to the predefined safety distance, then the whole resolution maneuver is safe.

We define $\left(S_{i j}^{k}\right)_{\min }(t)$ to be the minimum distance between aircraft $i$ and aircraft $j$ over the first half path if $k=1$ and over the second half path if $k=2$. In order to make multiple-aircraft conflict resolution safe, the following inequality is satisfied:

$$
\left(S_{i j}^{k}\right)_{\text {min }}(t) \geq R \text { for } t \in \begin{cases}{\left[0, \frac{T_{f}}{2}\right]} & \text { if } k=1 \\ \left(\frac{T_{f}}{2}, T_{f}\right] & \text { if } k=2\end{cases}
$$

where ij $\in\{12,23,13, \cdots,(N-1) N\}$, which leads to $N(N-1)$ safety conditions. For example, if we consider a three-aircraft conflict case $(N=$ $3)$, then there are six conditions for safe resolution. Condition $1\left(C_{1}\right)$ is $\left(S_{12}^{1}\right)_{\min }(t) \geq R$ and Condition $2\left(C_{2}\right)$ is $\left(S_{12}^{2}\right)_{\min }(t) \geq R$. Condition 3 $\left(C_{3}\right)$ and Condition $4\left(C_{4}\right)$ for aircraft 2 and aircraft 3 , and Condition 5 $\left(C_{5}\right)$ and Condition $6\left(C_{6}\right)$ for aircraft 1 and aircraft 3 can be obtained in the same way. We claim that the inequality in equation (5) for $i j \in$ $\{12,23,13, \cdots,(N-1) N\}$ comprise necessary and sufficient conditions for safety of multiple-aircraft conflict resolution for an exact conflict.

Lemma 1 There are no conflicts over $t \in\left[0, T_{f}\right]$ for an exact conflict case if and only if $\left(C_{1} \wedge C_{2}\right) \wedge\left(C_{3} \wedge C_{4}\right) \wedge \cdots \wedge\left(C_{N(N-1)-1} \wedge C_{N(N-1)}\right)$.

Proof: See Appendix A.

We compute the minimum distance over the first half path and over the second half path sequentially. From the geometry of the conflict resolution 
maneuver, the distance between aircraft along each straight path is a differentiable function of $t$, thus the derivative of the distance between aircraft with respect to time is always well defined and finite in these regions. The minimum distance can be obtained by setting the derivative of the distance squared between aircraft with respect to time to zero:

$$
\frac{d S_{i j}^{1}(t)}{d t}=0 \Rightarrow t_{\min }=-\frac{a b+c e}{a^{2}+c^{2}}
$$

After algebraic manipulation, it may be shown that equation (6) reduces to a very simple relation between heading change and the time at which the minimum distance occurs: $t_{\min }=T \cos u_{i}$. Substituting $t_{\text {min }}$ into equation (3), the safety condition between aircraft $i$ and aircraft $j$ over the first half path becomes

$$
S_{i j}^{1}\left(t_{\text {min }}\right)=\left(a^{2}+c^{2}\right)\left(b^{2}+e^{2}-R^{2}\right)-(a b+c e)^{2} \geq 0
$$

Equation (7) can be rewritten as:

$$
b^{2}+e^{2}-\frac{(a b+c e)^{2}}{a^{2}+c^{2}} \geq R^{2}
$$

where

$$
\begin{aligned}
b^{2}+e^{2} & =\left(r_{i} \cos \theta_{i}-r_{j} \cos \theta_{j}\right)^{2}+\left(r_{i} \sin \theta_{i}-r_{j} \sin \theta_{j}\right)^{2} \\
& =r_{i}^{2}+r_{j}^{2}-2 r_{i} r_{j} \cos \left(\theta_{i}-\theta_{j}\right) \\
a^{2}+c^{2} & =\left(v_{i} \cos \phi_{i}-v_{j} \cos \phi_{j}\right)^{2}+\left(v_{i} \sin \phi_{i}-v_{j} \sin \phi_{j}\right)^{2} \\
& =\frac{1}{T^{2}}\left[r_{i}^{2}+r_{j}^{2}-2 r_{i} r_{j} \cos \left(\theta_{i}-\theta_{j}\right)\right] \\
a b+c e & =\left(v_{i} \cos \phi_{i}-v_{j} \cos \phi_{j}\right)\left(r_{i} \cos \theta_{i}-r_{j} \cos \theta_{j}\right)+\left(v_{i} \sin \phi_{i}-v_{j} \sin \phi_{j}\right)\left(r_{i} \sin \theta_{i}-r_{j} \sin \theta_{j}\right) \\
& =-\frac{\cos u}{T}\left[r_{i}^{2}+r_{j}^{2}-2 r_{i} r_{j} \cos \left(\theta_{i}-\theta_{j}\right)\right]
\end{aligned}
$$

Since the heading changes of all aircraft are assumed to be the same $u:=$ $u_{i}=u_{j}$ for all $i, j=1,2, \cdots, N$, the safety condition in equation (8) can be simplified as

$$
\sin ^{2} u \geq \frac{R^{2}}{r_{i}^{2}+r_{j}^{2}-2 r_{i} r_{j} \cos \left(\theta_{i}-\theta_{j}\right)}
$$

where $i, j=1,2, \cdots, N, i \neq j$. 
Now, we consider the second half path. Similar to the first half path, the minimum distance occurs at

$$
\frac{d S_{i j}^{2}(t)}{d t}=0
$$

The same procedure as previous is taken, and the safety condition between aircraft $i$ and aircraft $j$ over the second half path is identical to the condition in equation (9) for the first half path. This is expected from the geometry of the resolution maneuvers into which the conflict resolution problem is cast because heading changes of all aircraft are the same and aircraft change their headings at the same time.

From equation (9), we see that the magnitude of the heading change required for conflict resolution is inversely proportional to the aircraft's distance to a conflict point and to the minimum relative angular distance among aircraft. This result coincides with the intuition that if aircraft are far apart from each other, then a small heading change is enough to resolve a conflict but if aircraft are close, then a bigger heading change is needed for safe separation. Equation (9) represents a closed-form analytic solution for the heading change required of all aircraft to resolve the conflict. The minimum heading change, $u_{m i n}$, can be obtained from equation (9) for a given initial configuration and known parameters. Since both $\pm u$ satisfy equation (9), we resolve this turn ambiguity for exact conflicts by restricting the control input to be such that $0^{\circ}<u<90^{\circ}$. Equation (9) (with turn ambiguity resolved) is simple and deterministic; and can be used to design a protocol for multiple-aircraft conflict resolution which can be implemented in both airborne systems and ground ATM systems, and also easily implemented for real-time applications because its computing time is negligible. No other currently available multiple-aircraft conflict resolution algorithm has all of these properties. The design of such a protocol for exact and inexact conflicts is the subject of the next two sections. 


\section{Derivation of a protocol for multiple-aircraft con- flict resolution for an exact conflict}

In this section, we construct a finite partition of the airspace around the conflict, and using our analytic solution in equation (9), we derive a protocol for resolving the worst-case conflict within each partition, where we define the worst-case conflict to be that configuration in which the required heading change to resolve the conflict is maximized. Thus, for each partition, the worst-case conflict is computed and the safe heading change which causes the minimum deviation from the desired trajectories of each aircraft is computed for this worst case. From equation (9), the minimum heading change which resolves a conflict is achieved when the denominator in the right-hand side is minimum, i.e. when $r_{i}=r_{j}=r_{\text {min }}$ and $\left|\theta_{i}-\theta_{j}\right|=\delta \theta_{\text {min }}$ :

$$
u_{\text {min }}=\sin ^{-1}\left(\frac{R}{r_{\min } \sqrt{2\left(1-\cos \delta \theta_{\text {min }}\right)}}\right)
$$

In this paper, we use parameters from the data of a B747 which cruises with Mach 0.8 at 40,000 ft altitude [5]. We set $r_{\min }=130 \mathrm{~nm}$ and $r_{\max }=170 \mathrm{~nm}$, though these are general and may be altered. All initial configurations are assumed to be safe.

For clarity, we derive the protocol below for a three-aircraft conflict, however, as we shall show, our method is general to $N$-aircraft conflicts. For a three-aircraft conflict, there are two possible worst cases as shown in Figure 4-(a). One is the case in which all three aircraft are initially at $r_{\text {min }}$ and separated by $R$. The other is the case in which all three aircraft are initially located on a line and separated by $R$. However, these two cases become unsafe instantaneously, and thus violate the assumption that a conflict occurs at the origin. To remedy these pathological situations, more restrictions must be imposed on the initial conditions: the restriction we impose is that the initial minimum distance between aircraft is not less than $3 R$. In fact, the initial minimum distance between aircraft can be any value greater than $R$, the reason we choose $3 R$ is to avoid large heading changes $\left(\geq 20^{\circ}\right)$ while at the same time making the restriction not overly conservative. Then, the two possible worst cases are: 


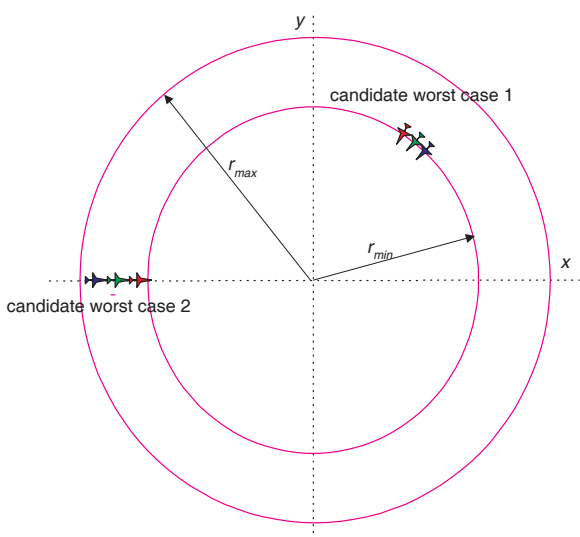

(a) Two possible worst cases.

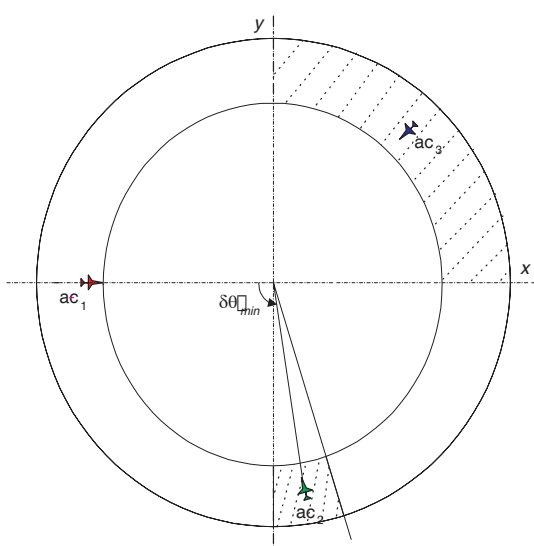

(b) Possible conflict configuration in Case 1 : $90^{\circ} \leq \delta \theta_{\min } \leq$ $120^{\circ}$.

Figure 4: Airspace partition.

- candidate worst case 1: $r_{1}=r_{2}=r_{\min }$ and $\delta \theta_{\min }=0.1154$

$\Rightarrow r_{\min } \sqrt{2\left(1-\cos \delta \theta_{\min }\right)}=224.8104$

- candidate worst case $2: r_{1}=r_{\min }, r_{2}=r_{\min }+3 R$, and $\delta \theta_{\min }=0$

$\Rightarrow r_{1}^{2}+r_{2}^{2}-2 r_{1} r_{2} \cos \delta \theta_{\min }=225$

Since "candidate worst case 1" results in a smaller denominator in equation (11) and thus in a maximum safe heading change, this gives the true worst case. Therefore, a protocol for the worst case is:

$$
u \geq 0.3400=19.4798^{\circ}
$$

This is an interesting result: it indicates that a triangular deviation maneuver as shown in Figure 3, with $u \geq 19.5^{\circ}$ for all aircraft, will resolve any conflict for any number of aircraft, as long as at the start of the maneuver, the radial positions of all aircraft are between $130 \mathrm{~nm}$ and $170 \mathrm{~nm}$, and the initial minimum distance between aircraft is not less than $3 R$. However, this result is overly conservative as it was computed using the worst case data. 
Thus, consider the following partition: we divide the airspace in the angular direction around the conflict point in order to alleviate conservativeness. Consider, for example, six different cases corresponding to the minimum relative angle between any pair of aircraft:

- Case 1: $90^{\circ} \leq \delta \theta_{\min } \leq 120^{\circ}$

- Case 2: $60^{\circ} \leq \delta \theta_{\min }<90^{\circ}$

- Case 3: $45^{\circ} \leq \delta \theta_{\min }<60^{\circ}$

- Case 4: $30^{\circ} \leq \delta \theta_{\min }<45^{\circ}$

- Case 5: $10^{\circ} \leq \delta \theta_{\min }<30^{\circ}$

- Case 6: $0^{\circ} \leq \delta \theta_{\min }<10^{\circ}$

where the minimum relative angle between aircraft is defined: $\delta \theta_{\min }=$ $\min \left\{\left|\theta_{i}-\theta_{j}\right| \mid i, j=1,2, \cdots, N\right.$, and $\left.i \neq j\right\}$ as shown in Figure 4-(b) for a three-aircraft conflict. For each of these cases, we compute the worst-case conflict and the safe heading change which causes the minimum deviation from the desired trajectories of each aircraft.

Consider, for example, Case 1, in which without loss of generality, the relative angle between aircraft 1 and aircraft 2 is assumed to be minimum. Place aircraft 1 on the negative $x$-axis as shown in Figure 4 -(b), such that the conflict occurs at the origin. To satisfy the hypothesis of Case 1, aircraft 2 should be located inside the shaded region, $270^{\circ} \leq \theta_{2} \leq 300^{\circ}$ (since $\delta \theta_{\text {min }}$ is $90^{\circ}$ ) and aircraft 3 could be located somewhere inside the shaded region, $0^{\circ} \leq \theta_{3} \leq 90^{\circ}$, as shown in Figure 4-(b). Here, the shaded region which contains aircraft 3 is the largest possible one. Indeed, if aircraft 2 lies on the negative $y$-axis, then aircraft 3 could lie on the positive $x$-axis, and the hypothesis of Case 1 would still be satisfied. The minimum safe heading change can be obtained by substituting $r_{\min }=130 \mathrm{~nm}$ and $\delta \theta_{\min }=90^{\circ}$ into equation (11). This procedure can be similarly applied to the other five cases (where the protocol for Case 6 is the same as equation (12) derived earlier), leading to the following protocol: 


\section{Multiple-aircraft protocol in the case of exact conflict}

- Case 1: $90^{\circ} \leq \delta \theta_{\min } \leq 120^{\circ} \Rightarrow u \geq 0.0272=1.5584^{\circ}$

- Case 2: $60^{\circ} \leq \delta \theta_{\min }<90^{\circ} \Rightarrow u \geq 0.0385=2.2048^{\circ}$

- Case 3: $45^{\circ} \leq \delta \theta_{\min }<60^{\circ} \Rightarrow u \geq 0.0503=2.8805^{\circ}$

- Case 4: $30^{\circ} \leq \delta \theta_{\min }<45^{\circ} \Rightarrow u \geq 0.0744=4.2628^{\circ}$

- Case 5: $10^{\circ} \leq \delta \theta_{\min }<30^{\circ} \Rightarrow u \geq 0.2225=12.7471^{\circ}$

- Case 6: $0^{\circ} \leq \delta \theta_{\min }<10^{\circ} \quad \Rightarrow \quad u \geq 0.3400=19.4798^{\circ}$

If we consider an $\mathrm{N}$-aircraft conflict in which the minimum relative angular position among $N$ aircraft is, for example, $50^{\circ}$, then heading change $u=2.8805^{\circ}$ guarantees safety of the $N$-aircraft conflict resolution. In general, we consider the case in which each aircraft has the authority to choose its own airspace partition. Since the finer the airspace partition, the smaller the deviation, it is reasonable that each aircraft may select its own airspace partition according to its on-board computing ability and aerodynamic performance. Then, the resolution procedure is as follows:

\section{Algorithm 1 (Conflict resolution procedure in the case of exact conflict)}

We assume that each aircraft has access to the other aircraft's position, velocity, and heading, and thus detects a conflict:

1. Each aircraft chooses a partition, computes its own heading change according to this partition, and broadcasts this heading change $u_{i}$ to all other aircraft;

2. Among all heading changes broadcast including its own each aircraft chooses the smallest (i.e. $u=\min _{i}\left|u_{i}\right|$, for $i=1,2, \cdots, N$.), and follows this protocol.

Since each aircraft has the authority to choose its own airspace partition and to compute its own resolution maneuver from Algorithm 1, our method is a decentralized conflict resolution method. 
Proposition 1 Algorithm 1 guarantees safety for a multiple-aircraft exact conflict.

Proof: Since each aircraft's heading change $u_{i}$ satisfies equation (9), from Lemma 1, Step 1 of Algorithm 1 is safe. Since all heading changes $u_{i}$ chosen in Step 2 of Algorithm 1 are safe, the smallest $u$ guarantees safety. Algorithm 1 guarantees safety of $N$-aircraft conflicts because Lemma 1 and the safety condition in equation (9) do not depend on the number of aircraft involved in the conflict.

\section{Simulation results}

Simulation and validation are performed with a dynamic aircraft model under linear control using the data from Bryson [5] of a B747 which cruises with Mach 0.8 at 40,000 ft altitude, presented in Appendix B. If the resolution procedure designed above is applied to an arbitrary initial configuration (which has a conflict point at the origin), the conflict-free resolution maneuvers are obtained as shown in Figure 5 for a three-aircraft conflict (for all six cases in the example above) and Figure 6 for a ten-aircraft conflict. The ten-aircraft scenario converging to a conflict point is motivated from Bilimoria [4], Frazzoli et al.[13], and Hoekstra et al[17]. Since the computing time for Algorithm 1 is negligible because the protocol is based on a simple geometric inequality in equation (9), it can be used for real-time airborne applications.

\section{Derivation of a protocol for multiple-aircraft con- flict resolution for an inexact conflict}

The exact conflict case is unrealistic and rarely occurs, yet the methodology of the previous sections may be extended to the inexact case. In this section, we relax the exact assumption to consider the case in which the conflict points of a multiple aircraft conflict do not coincide. For $N$-aircraft conflicts, there are at most $\frac{N(N-1)}{2}$ original conflict points. We consider two cases: one is a perturbation of an exact conflict in which all conflict points occur 

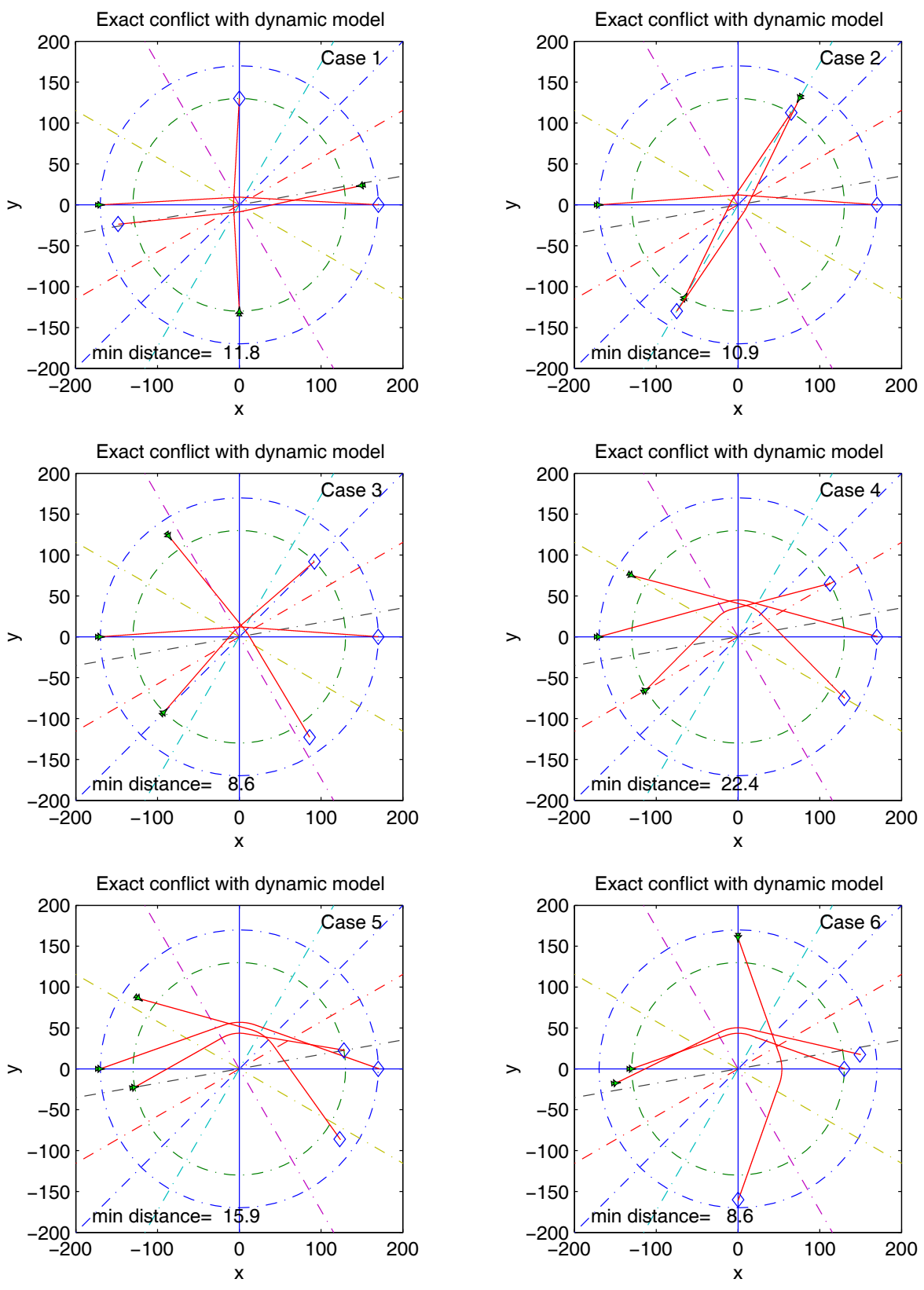

Figure 5: Protocol-based conflict resolution for three aircraft $(\mathrm{nm})$. 


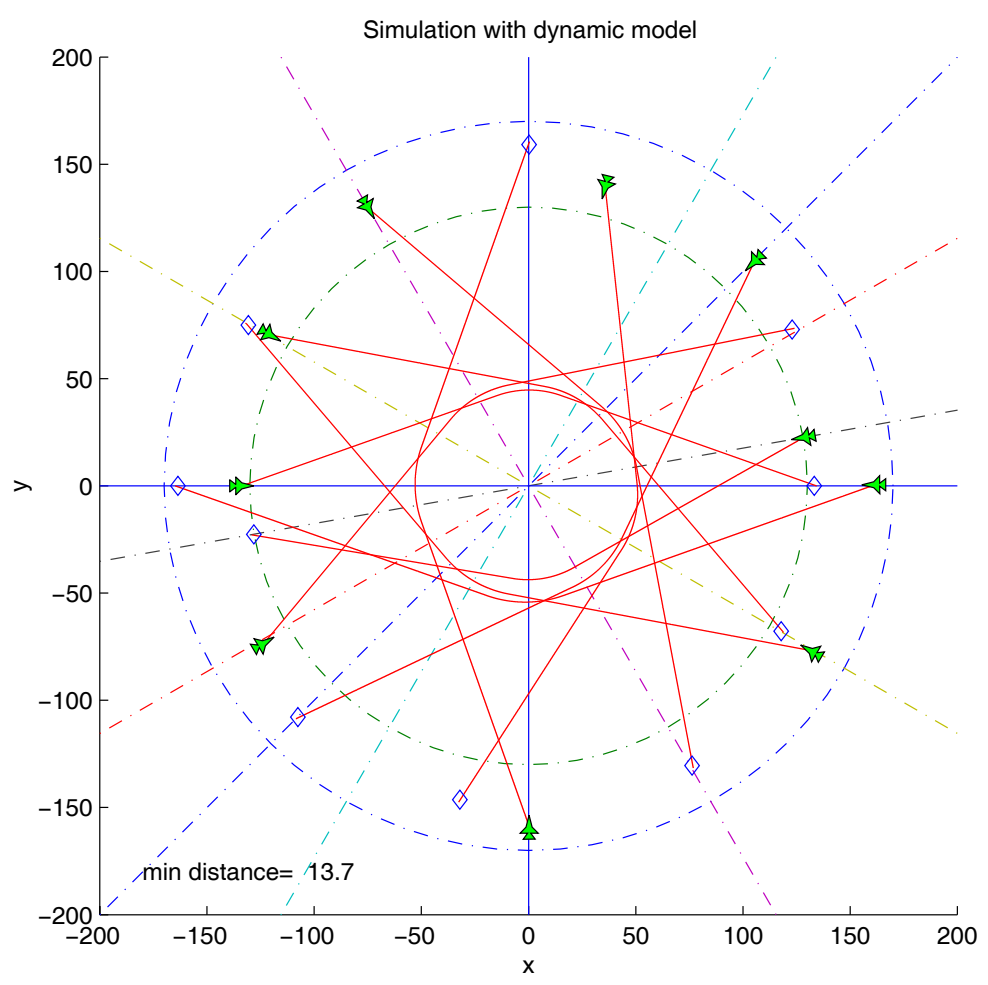

Figure 6: Protocol-based conflict resolution for ten aircraft $(\mathrm{nm})$. 
within a circle whose diameter is $R$, the required separation distance between aircraft. The other case we call a general inexact conflict, in which conflict points lie arbitrarily, not necessary in circle of radius $R$.

\subsection{Perturbation of an exact conflict}

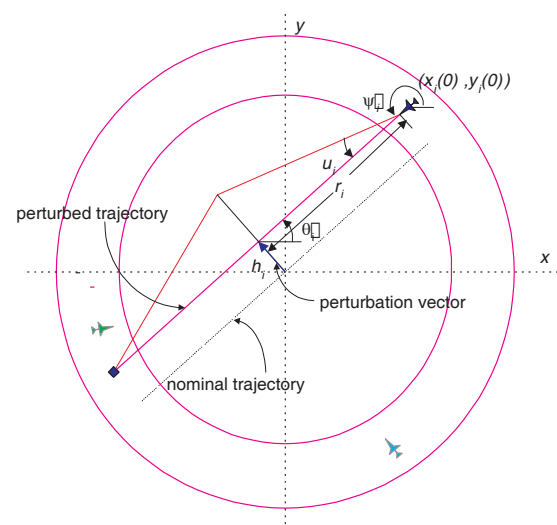

(a) Perturbation case; conflict points lie within a circle of diameter $R$.

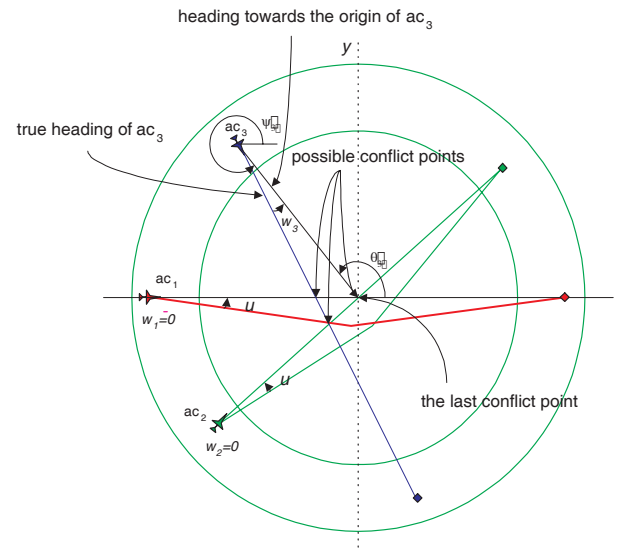

(b) General case; conflict points separated by distance greater than $R$.

Figure 7: Inexact conflict.

Consider first an inexact conflict which is a perturbation of an exact conflict, as shown in Figure 7-(a). The origin of the conflict resolution is chosen as the center of a circle of minimum radius which includes all conflict points. The nominal trajectory of aircraft $i$ can be described as the perturbation of a nominal trajectory which passes through the origin by a perturbation vector $\mathbf{h}_{\mathbf{i}}$ whose magnitude $h_{i}=\left\|\mathbf{h}_{\mathbf{i}}\right\|$ is assumed to be less than or equal to $\frac{R}{2}$. The initial position of aircraft $i$ is denoted as 
$\left(x_{i}(0), y_{i}(0)\right)$ such that

$$
\begin{aligned}
{\left[\begin{array}{l}
x_{i}(0) \\
y_{i}(0)
\end{array}\right] } & =\left[\begin{array}{c}
r_{i} \cos \theta_{i} \\
r_{i} \sin \theta_{i}
\end{array}\right]+\mathbf{h}_{\mathbf{i}} \\
& =\left[\begin{array}{c}
r_{i} \cos \theta_{i}+h_{i} \cos \left(\theta_{i}+90^{\circ}\right) \\
r_{i} \sin \theta_{i}+h_{i} \sin \left(\theta_{i}+90^{\circ}\right)
\end{array}\right]
\end{aligned}
$$

where $i=1,2, \cdots, N$.

The safety condition for this case can be obtained by following the same procedure in Section 4. For notational simplicity, we define for aircraft $i$ and aircraft $j$ :

$H:=r_{i}^{2}+r_{j}^{2}-2 r_{i} r_{j} \cos \left(\theta_{i}-\theta_{j}\right), \quad L:=h_{i}^{2}+h_{j}^{2}-2 h_{i} h_{j} \cos \left(\theta_{i}-\theta_{j}\right)-2\left(r_{i} h_{j}-\right.$ $\left.r_{j} h_{i}\right) \sin \left(\theta_{i}-\theta_{j}\right)$

$M:=-H+\left(r_{i} h_{j}-r_{j} h_{i}\right) \sin \left(\theta_{i}-\theta_{j}\right), \quad F:=-\left(r_{i} h_{i}+r_{j} h_{j}\right)+\left(r_{j} h_{j}+\right.$ $\left.r_{j} h_{i}\right) \cos \left(\theta_{i}-\theta_{j}\right)$

Then, the safety condition for aircraft $i$ and aircraft $j$ is:

$$
H+L-\frac{1}{H}(M \cos u+F \sin u)^{2} \geq R^{2}
$$

where $i, j=1,2, \cdots, N$. Equation (14) must be solved numerically. If we use the same six partitions as in Section 5 and $h_{i} \in\left[0, \frac{R}{2}\right]$, the protocol for an inexact perturbation case is as follows:

\section{Multiple-aircraft protocol in the case of inexact conflict (Per- turbation case)}

- Case 1: $\quad 90^{\circ} \leq \delta \theta_{\min } \leq 120^{\circ} \Rightarrow u \geq 0.0552=3.1627^{\circ}$

- Case 2: $60^{\circ} \leq \delta \theta_{\min }<90^{\circ} \Rightarrow u \geq 0.0608=3.4836^{\circ}$

- Case 3: $45^{\circ} \leq \delta \theta_{\min }<60^{\circ} \Rightarrow u \geq 0.0711=4.0737^{\circ}$

- Case 4: $30^{\circ} \leq \delta \theta_{\min }<45^{\circ} \Rightarrow u \geq 0.0943=5.4030^{\circ}$

- Case 5: $10^{\circ} \leq \delta \theta_{\min }<30^{\circ} \Rightarrow u \geq 0.2867=16.4267^{\circ}$

- Case 6: $0^{\circ} \leq \delta \theta_{\min }<10^{\circ} \Rightarrow u \geq 0.5235=29.9943^{\circ}$

The algorithm for conflict resolution in this case is the same as that for an exact conflict (Algorithm 1), with slightly larger heading changes which may be computed offline (and stored) for given partitions. 


\subsection{General inexact conflict}

We now generalize to the case of a true inexact conflict; that is, that the set of aircraft comes into conflict at points separated by a distance greater than $R$. We generalize our protocol so that only the first segment of a resolution maneuver contributes to safe separation (the case in which $k=1$ in equation (5)). The second segment of the resolution maneuver is used in order to return to the nominal trajectory. For example, if we consider the three-aircraft conflict, Condition $1\left(C_{1}\right)$ is $\left(S_{12}^{1}\right)_{\min }(t) \geq R$, Condition $2\left(C_{2}\right)$ is $\left(S_{23}^{1}\right)_{\min }(t) \geq R$ and Condition $3\left(C_{3}\right)$ is $\left(S_{13}^{1}\right)_{\min }(t) \geq R$ where $t \in\left[0, \frac{T_{f}}{2}\right]$. For $N$-aircraft conflicts, there are at most $\frac{N(N-1)}{2}$ conflict points.

If the point at which the last conflict among all conflicts occurs is chosen as the origin of our reference frame, and thus the midpoint of each aircraft's nominal trajectory segment for resolution purposes, then the other possible conflict points are on the first segments of each aircraft's nominal trajectory. In this case, only the first segment has to be considered in order to derive a safety condition $[13,1]$. The three-aircraft example is illustrated in Figure 7-(b).

Our strategy for solving this general conflict will be to first transform the problem to that of an exact conflict, and then to use the solution derived in Section 5 for an exact conflict to resolve the transformed problem. We then map the resolution maneuver back to the coordinates of the general conflict, and the general resolution results. The proof of safety follows from the fact that the exact resolution solves the transformed problem, and that the mapping between the original and transformed problem is unique.

Thus, we introduce new variables $w_{i}$, defined as the heading difference between the true heading of aircraft $i$ and the origin of our reference frame, as shown in Figure 7-(b) for aircraft 3. Here, we define the counter-clockwise direction as positive, thus $\operatorname{sign}\left(w_{3}\right)=-1$, where the sign function is defined as:

$$
\operatorname{sign}(x)= \begin{cases}1 & \text { if } x \geq 0 \\ -1 & \text { if } x<0\end{cases}
$$

Now suppose that aircraft $i$ were not involved in the conflict at the origin; if we adjust the velocity of aircraft $i$ such that $v_{i}=\frac{r_{i}}{T}$ and we change its 
heading by $w_{i}$, then it would be involved in a conflict at the origin. If we performed this transformation for all aircraft not involved in conflict at the origin (at most $N-2$ aircraft), then the inexact conflict would become an exact conflict, and the required heading change $u$ for safe resolution of this exact conflict can be obtained from Algorithm 1. For clarity, we denote this $u$ as $u_{\text {exact }}$ in this section.

Now, define for aircraft $i$ and aircraft $j: \phi_{i}:=\psi_{i}-u, \bar{a}:=v_{i} \cos \phi_{i}-$ $v_{j} \cos \psi_{j}, \bar{b}:=r_{i} \cos \theta_{i}-r_{j} \cos \theta_{j}, \quad \bar{c}:=v_{i} \sin \phi_{i}-v_{j} \sin \psi_{j}, \quad \bar{e}:=r_{i} \sin \theta_{i}-$ $r_{j} \sin \theta_{j}$, where $i, j=1,2, \cdots, N$. Then, the distance squared between aircraft $i$ and aircraft $j$ is:

$$
\begin{aligned}
S_{i j}^{1}(t)= & \left(x_{i}(t)-x_{j}(t)\right)^{2}+\left(y_{i}(t)-y_{j}(t)\right)^{2} \\
= & {\left[\left(v_{i} \cos \phi_{i}-v_{j} \cos \psi_{j}\right) t+\left(r_{i} \cos \theta_{i}-r_{j} \cos \theta_{j}\right)\right]^{2} } \\
& +\left[\left(v_{i} \sin \phi_{i}-v_{j} \sin \psi_{j}\right) t+\left(r_{i} \sin \theta_{i}-r_{j} \sin \theta_{j}\right)\right]^{2} \\
= & (\bar{a} t+\bar{b})^{2}+(\bar{c} t+\bar{e})^{2}
\end{aligned}
$$

The safety condition between aircraft $i$ and aircraft $j$ becomes

$$
\bar{b}^{2}+\bar{e}^{2}-\frac{(\bar{a} \bar{b}+\bar{c} \bar{e})^{2}}{\bar{a}^{2}+\bar{c}^{2}} \geq R^{2}
$$

We define $w_{\max }=\max _{i}\left|w_{i}\right| \cdot \operatorname{sign}\left(w_{i}\right), i=1,2, \cdots, N$ : thus $w_{\max }$ has the maximum magnitude among $w_{i}$ and preserves sign. For example, in the case of Figure 7 -(b), $w_{1}=0, w_{2}=0$, and $w_{3}=\left|w_{3}\right| \operatorname{sign}\left(w_{3}\right)$, thus $w_{\max }=-w_{3}$. If we assumed that $u_{\text {exact }}=w_{\max }$ and follow the same procedure as in Section 4, the safety condition for a general inexact conflict would be:

$$
\sin ^{2} u_{\text {exact }} \geq \frac{R^{2}}{r_{1}^{2}+r_{3}^{2}-2 r_{1} r_{3} \cos \left(\theta_{1}-\theta_{3}\right)}
$$

which is identical to the safety condition for an exact conflict in equation (9). However, the assumption that $u_{\text {exact }}=w_{\max }$ may not necessarily be true. Thus, we consider the two cases $u_{\text {exact }}>\left|w_{\max }\right|$ and $u_{\text {exact }} \leq\left|w_{\max }\right|$ : if $u_{\text {exact }}>\left|w_{\max }\right|$, a safe heading change for a general inexact conflict must be $u_{\text {exact }}$ because $\left|w_{\max }\right|$ would not satisfy the safety condition in equation (17); if $u_{\text {exact }} \leq\left|w_{\max }\right|$, then both $u_{\text {exact }}$ and $w_{\max }$ satisfy equation (17). However, since the sign of the heading change of all aircraft should be the 
same according to our protocol, then $u$ must be set to $w_{\max }$. Therefore, a protocol $u$ for a general inexact conflict is:

$$
u= \begin{cases}u_{\text {exact }} \operatorname{sign}\left(w_{\text {max }}\right) & \text { if } u_{\text {exact }}>\left|w_{\text {max }}\right| \\ w_{\text {max }} & \text { if } u_{\text {exact }} \leq\left|w_{\text {max }}\right|\end{cases}
$$

Since this protocol $u$ is computed in the transformed frame, aircraft $i$ must actually change its heading by $u-w_{i}(i=1,2, \cdots, N)$. The protocol for a general inexact conflict is as follows:

Algorithm 2 (Multiple-aircraft protocol in the case of inexact conflict (general case))

New Protocol (heading change + velocity change)

For $i=1,2, \cdots, N$ :

1. Select the last conflict point among possible conflict points as the center of conflict resolution;

2. If aircraft $i$ is not involved in the conflict at the origin, adjust its velocity such that $v_{i}=\frac{r_{i}}{T}(\leftarrow$ new velocity. $)$;

3. Aircraft $i$ computes its own heading change $u_{\text {exact }}$ from Algorithm 1, and computes $w_{i}, w_{\max }$, and $u$;

4. Aircraft $i$ changes its heading by $u-w_{i}$.

Algorithm 2 may be implemented in a decentralized manner, since each aircraft recognizes a conflict situation and computes its own resolution maneuver autonomously.

Proposition 2 Algorithm 2 guarantees safety for the multiple-aircraft inexact conflict (general case).

Proof: Step $1 \sim$ Step 3 transform a general inexact conflict situation into an exact conflict, and the heading changes in Steps 3 and 4 satisfy the safety condition in equation (9). Therefore, from Lemma 1, the multiple-aircraft protocol in the case of a general inexact conflict guarantees safety. 


\subsection{Simulation results}

If the two protocols for an inexact conflict are applied to an arbitrary initial configuration which satisfies the given assumptions that are the same as those of an exact conflict, the conflict-free resolution maneuvers are obtained as shown in Figure 8 for the perturbation case, and Figure 9 for the general case. A symmetric encounter pattern for four-aircraft conflicts [17] and a scenario of two converging aircraft streams in Figure 8 and random encounter patterns for ten-aircraft conflicts in Figure 8 and Figure 9 are motivated from Frazzoli et al.[13].

Figure 9-(e) shows that if an aircraft lies within the airspace partition around the center of the conflict resolution, the aircraft must follow the protocol for safety even though the aircraft is not involved in any conflicts within the airspace partition. This fact that all aircraft take resolution maneuvers at the same time coincides with other multiple-aircraft resolution methodologies $[13,20,2]$. As in Section $3, r_{\min }$ and $r_{\max }$ are the radii of two concentric circular discs around the conflict. From the data of a B747 with Mach 8 at 40,000 ft, we used $r_{\min }=130 \mathrm{~nm}$ and $r_{\max }=170 \mathrm{~nm}$ throughout this paper. However, the simulation result with $r_{\min }=30 \mathrm{~nm}$ and $r_{\max }=50 \mathrm{~nm}$ in the airspace partition in Figure 9-(f) shows that our protocol is valid with respect to various airspace partitions with different $r_{\text {min }}$ and $r_{\text {max }}$, which is detailed in random simulation results in Appendix B. Appendix B presents the detail of validation with the dynamic aircraft model and random simulation results with different parameters such as lookahead time, initial minimum distance between aircraft, and the number of airspace partitions. Simulation results show that the computing time for Algorithm 2 is negligible. The reason is the protocol is based on a simple geometric inequality in equation (9) instead of computationally intensive algorithms such as randomized searches and numerical optimization.

\section{Robustness Analysis}

Demonstrating robustness of the protocol for multiple-aircraft conflict resolution is important; the protocol must guarantee safety against uncertainty 


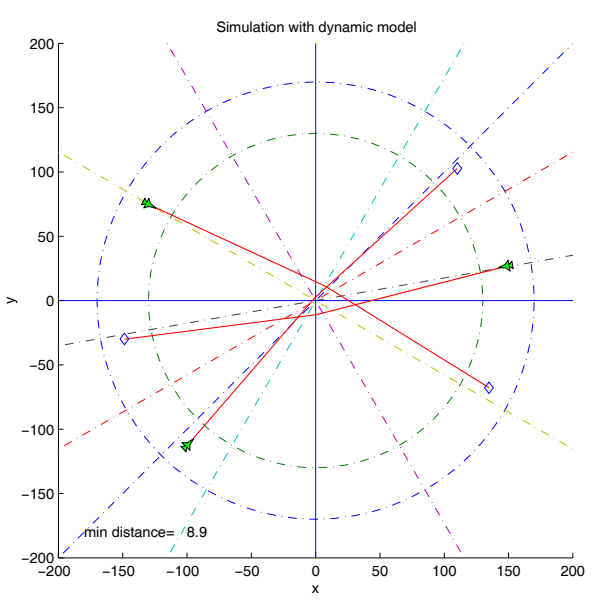

(a) For three aircraft.

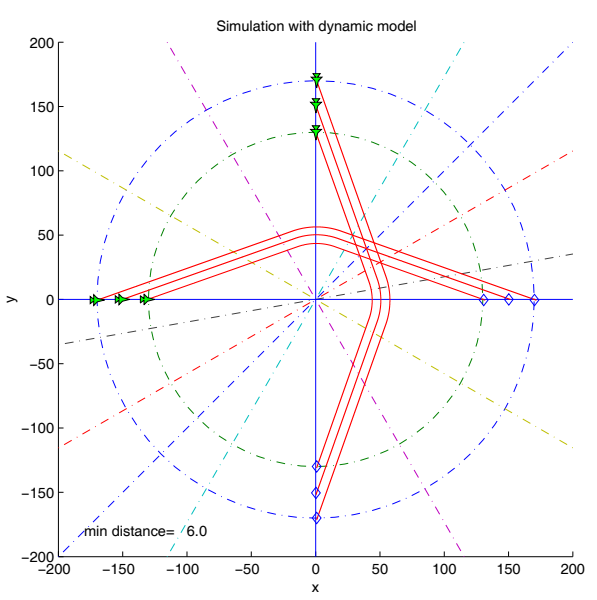

(c) For six aircraft.

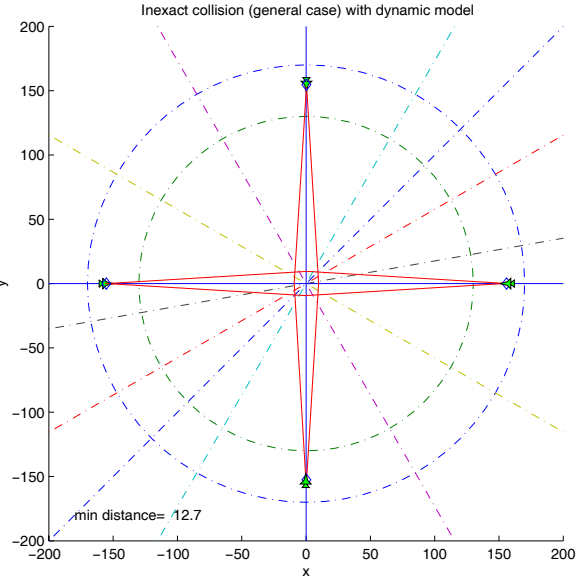

(b) For four aircraft.

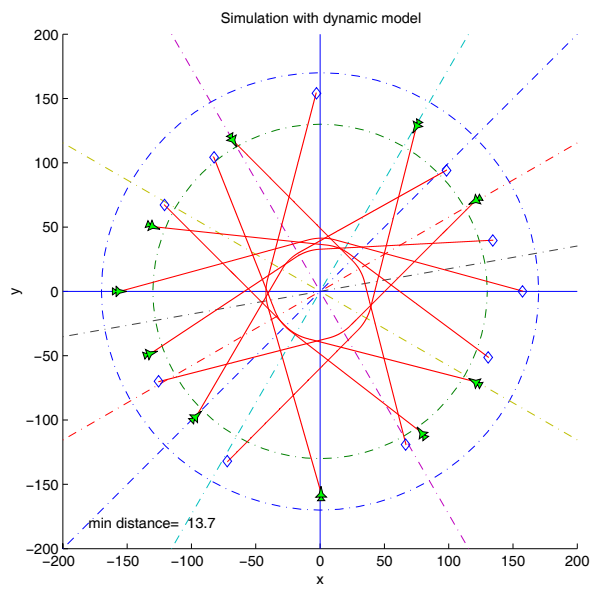

(d) For ten aircraft.

Figure 8: Inexact conflict (Perturbation case) $(\mathrm{nm})$. 


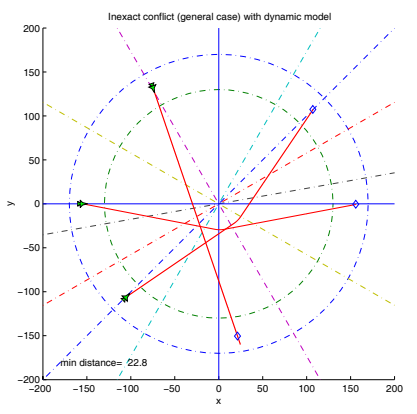

(a) For three aircraft.

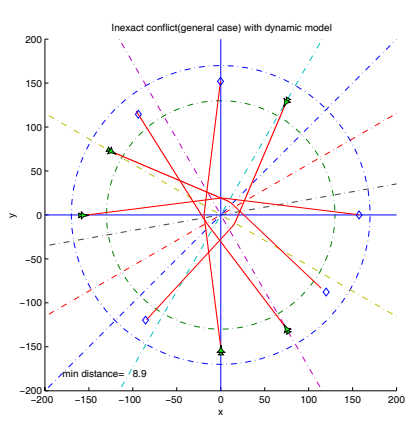

(c) For five aircraft.

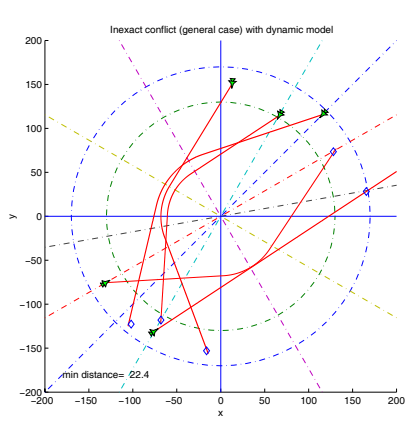

(e) For five aircraft with an aircraft not involved in any conflicts.

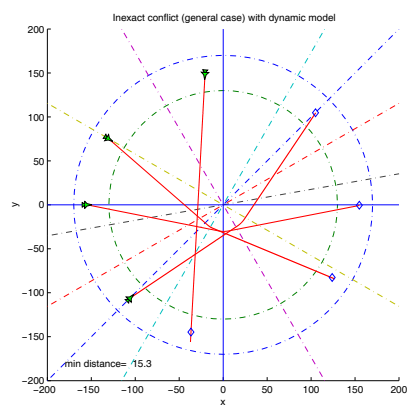

(b) For four aircraft.

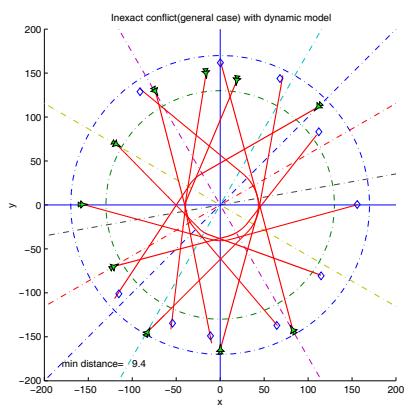

(d) For ten aircraft.

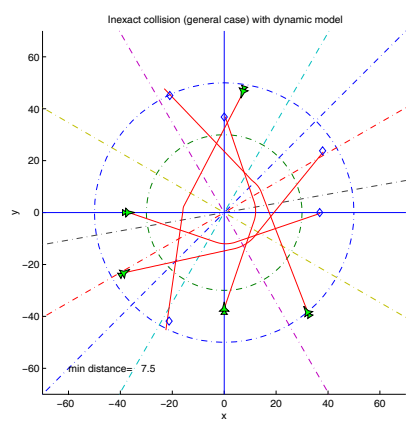

(f) For five aircraft with $r_{\min }=30 \mathrm{~nm}$ and $r_{\max }=50 \mathrm{~nm}$.

Figure 9: Inexact conflict (General case) $(\mathrm{nm})$. 
in the position, velocity, and heading of the aircraft. In the following, we show the protocol is robust with respect to uncertainty in sector boundary, to maneuver shape, and to asynchronous maneuvers for both exact and perturbed maneuvers.

\subsection{Robustness analysis with respect to sector boundaries}

Consider Figure 10-(a). We express sectors of allowable initial conditions for aircraft as $\left(r_{\min } \leq r \leq r_{\max }\right) \wedge\left(\theta_{\min } \leq \theta \leq \theta_{\max }\right)$, shown as a shaded region in Figure 10-(a). The uncertainties that are important for the protocol are those in $r_{\text {min }}$ and $\delta \theta_{\text {min }}$, as seen from equation (11). Uncertainty in $r_{\text {min }}$ is considered first. If $r_{\min }$ has $\pm 5 \%$ error, a new heading change $u_{i}$ can be obtained from equation (11) for both the exact conflict case and the general inexact conflict case, and from equation (14) for an inexact conflict perturbation case. For an exact conflict, $u_{i}$ should be:

$$
u_{i} \geq \sin ^{-1}\left(\frac{R}{0.95 r_{\min } \sqrt{2\left(1-\cos \delta \theta_{\min }\right)}}\right)
$$

If Case 1 is considered, $u_{i} \geq 0.02864$ which differs from the original heading change by $5.29 \%$. But since the heading change itself is small, the difference is only $0.0825^{\circ}$. For Case $5, u_{i} \geq 0.2344$ which differs from the original heading change by $5.35 \%$ and by $0.6818^{\circ}$. Similarly, for an inexact conflict perturbation case, $u_{i} \geq 0.0620$ for Case 1 which differs from the original heading change by $0.3896^{\circ}$ and for Case $5, u \geq 0.3070$ which differs from the original heading change by $1.1631^{\circ}$. From this analysis, we know that the protocols for multiple-aircraft conflict resolution are robust to uncertainty in $r_{\text {min }}$. To make conflict resolution in both Algorithm 1 and Algorithm 2 safe with respect to uncertainty in $r_{\text {min }}$, a new $r_{\text {min }}$ which takes into account this uncertainty should be used when each aircraft computes its own heading change $u_{i}$.

Now, consider uncertainty in $\delta \theta_{\text {min }}$. Since the protocol is derived from the worst case in each category, the control input, $u_{i}$, is the minimum heading change which can resolve conflicts for a given category. If the aircraft position is uncertain due to sensor errors, it can be represented by a region, 
usually a circle or an ellipse. If this region crosses the sector boundary as shown in Figure 10-(a), the larger $u_{i}$ must be chosen for safety. For example, in Figure 10-(a), if there is no uncertainty in the position of aircraft 2, then this configuration corresponds to Case 1. Thus, the protocol for an inexact conflict perturbation case is $u_{i}=0.0552$. However, if there is uncertainty in the position of aircraft 2 , then the protocol for an inexact conflict perturbation case must be that for Case 2, i.e. $u_{i}=0.0608$ rather than $u_{i}=0.0552$. Similarly, the larger $u_{i}$ must be chosen for safety for an exact conflict case. Then, the protocols for multiple-aircraft conflict resolution are robust to uncertainty in $\delta \theta_{\min }$.

\subsection{Robustness analysis with respect to circular arc turns}

We assumed instantaneous heading changes when deriving the protocol for multiple-aircraft conflict resolution, impossible in a real situation. Thus, we consider robustness with respect to replacing corners by circular arc turns. Again, without loss of generality, we consider three-aircraft conflicts. In Figure 10-(b), all three aircraft follow the conflict resolution paths: consider aircraft 1 and aircraft 2 . Aircraft 1 and aircraft 2 reach $q_{1}$ and $q_{2}$ respectively at the same time. On the circular arcs, the two aircraft move with the same angular velocity, $\omega$. This can be easily shown. The distance $l_{1}$ in Figure 10 -(b) is

$$
l_{1}=v_{1} T \cos u_{1}
$$

Thus, the angular velocity of aircraft 1 is:

$$
\omega_{1}=\frac{v_{1}}{l_{1}}=\frac{v_{1}}{v_{1} T \sin u_{1}}=\frac{1}{T \sin u_{1}}=: \omega
$$

From equation (20), the angular velocity of aircraft 1 depends on $T$ and $u_{1}$ only. Since $u_{1}=u_{2}=u_{3}$, the angular velocities of all three aircraft are the same.

To show robustness of the protocol, we need only to show now that the maneuvers are safe when the aircraft are on the circular arc paths. Of course, the worst case occurs when the distance between aircraft on the circular paths is minimum. From the geometry and the fact that all three 


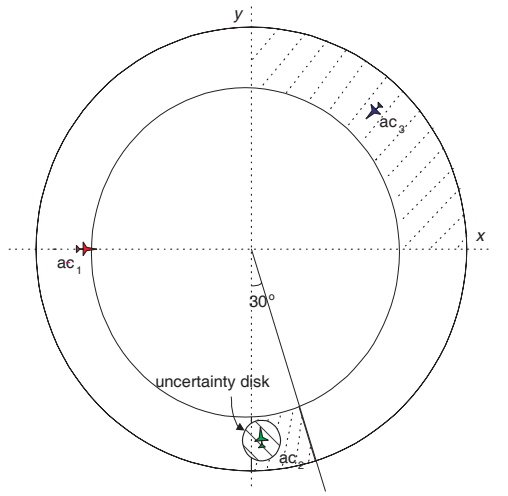

(a) Airspace sectors and parameter uncertainty for Case 1.

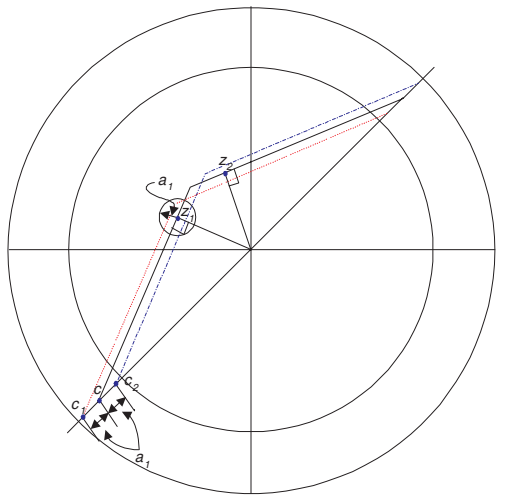

(c) Asynchronous maneuver at a starting point.

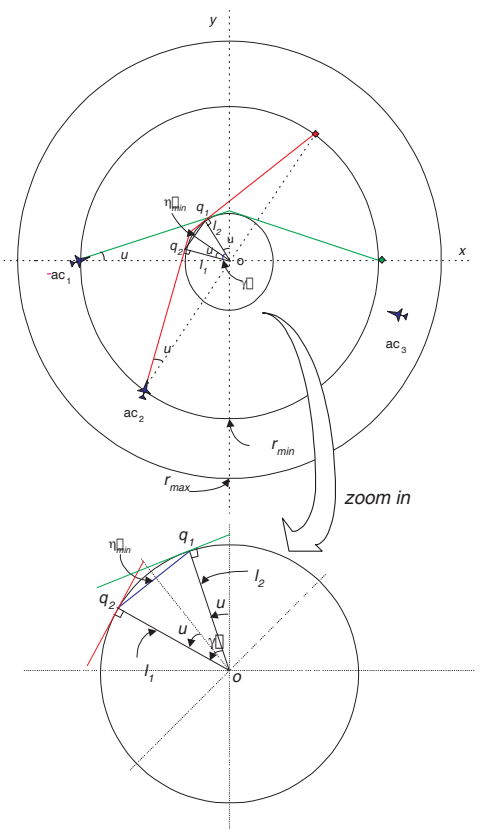

(b) Robust analysis to smoothing corners.

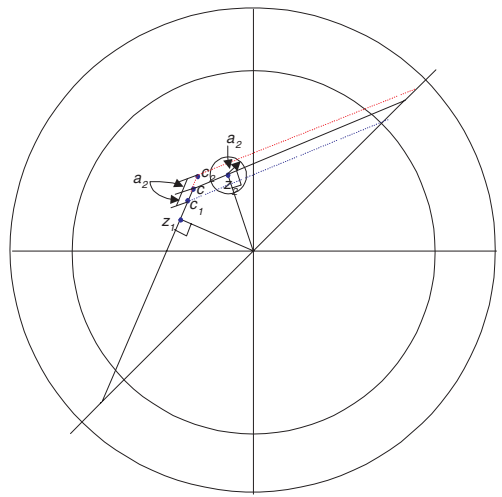

(d) Asynchronous maneuver at a new waypoint.

Figure 10: Robustness analysis. 
aircraft have the same angular velocity during the resolution maneuver, the worst case is achieved between a point $q_{1}$ and a point $q_{2}$ when aircraft 1 and aircraft 2 are both on $r_{\min }$ and the relative angle between aircraft 1 and aircraft 2 is $\delta \theta_{\text {min }}$. The minimum distance between aircraft on the circular arcs from Figure 10-(b) is:

$$
\eta_{\text {min }}^{2}=2 r_{\text {min }}^{2}(1-\cos \gamma)
$$

where $\gamma$ is $\delta \theta_{\text {min }}$. Therefore, the minimum distance on the circular arcs is:

$$
\eta_{\min }=r_{\min } \sqrt{2\left(1-\cos \delta \theta_{\min }\right)}
$$

If $\eta_{\text {min }} \geq R$ for all possible conflict cases over the circular arcs, the resolution maneuvers are safe, i.e. the protocol for three-aircraft conflict resolution is robust to smoothing corners. If equation (22) is applied to all six cases, the results are:

- Case 1: $\quad 90^{\circ} \leq \delta \theta_{\min } \leq 120^{\circ} \Rightarrow \eta_{\min }=5.0004>R \Rightarrow$ safe

- Case 2: $\quad 60^{\circ} \leq \delta \theta_{\min }<90^{\circ} \quad \Rightarrow \quad \eta_{\min }=5.0038>R \Rightarrow$ safe

- Case 3: $45^{\circ} \leq \delta \theta_{\min }<60^{\circ} \quad \Rightarrow \quad \eta_{\min }=5.0026>R \Rightarrow$ safe

- Case 4: $30^{\circ} \leq \delta \theta_{\min }<45^{\circ} \Rightarrow \eta_{\min }=5.0032>R \Rightarrow$ safe

- Case 5: $10^{\circ} \leq \delta \theta_{\min }<30^{\circ} \quad \Rightarrow \quad \eta_{\min }=5.0005>R \Rightarrow$ safe

- Case 6: $0^{\circ} \leq \delta \theta_{\min }<10^{\circ} \quad \Rightarrow \quad \eta_{\min }=5.0002>R \Rightarrow$ safe

This shows that the protocol for an exact conflict is robust to replacing corners by circular arc turns. Since the protocol for a general inexact conflict is based on the protocol for an exact conflict, robustness of the protocol for a general inexact conflict to replacing corners by circular arc turns can be easily shown by following the same procedure for an exact conflict. Since angular velocities of all aircraft are the same, the minimum distance between aircraft remains same after replacing corners by circular arcs. Thus, the protocol for an inexact conflict perturbation case is also robust to replacing corners by circular arcs. 


\subsection{Robustness analysis with respect to asynchronous ma- neuvers.}

We assumed synchronous maneuvers, in which all aircraft change their heading at the same time, when deriving the protocol for multiple-aircraft conflict resolution. Here, we show that our protocol is robust with respect to asynchronous maneuvers.

First, consider the case in which aircraft initiate their resolution maneuver earlier or later than the exact resolution starting time, as shown in Figure 10-(c). In Figure 10-(c), $c$ represents an exact resolution starting point. If an aircraft initiates its resolution maneuver earlier or later than the exact resolution starting point, $c_{1}$ and $c_{2}$ represent a real resolution starting point respectively and $a_{1}$ is the distance between $c$ and $c_{1}$ (or $c_{2}$ ). If the aircraft's velocity is $v$, then the time discrepancy $\tau_{1}$ between the exact resolution starting time and a real resolution starting time at the resolution starting point is defined as $\tau_{1}=\frac{a_{1}}{v}$. From equation (6), the minimum distance among aircraft occurs at $z_{1}$ on the first segment and at $z_{2}$ on the second segment. Due to asynchronous maneuvers, the minimum distance can be changed by $a_{1}$ at $z_{1}$ and $z_{2}$. We use an uncertainty disk with radius $a_{1}$ at the $z_{1}$ and $z_{2}$ to accommodate this change in the minimum distance [19]. Then, for safety, a new predefined safety distance $R_{\text {safe }}$ should be

$$
R_{\text {safe }}=R+v \tau_{1}
$$

We consider the case in which aircraft change their heading before or after reaching a new waypoint $c$ as shown Figure 10-(d). In Figure 10-(d), $a_{2}$ is the distance between $c$ and $c_{1}$ (or $c_{2}$ ) and the time discrepancy $\tau_{2}$ at the new waypoint is defined as $\tau_{2}=\frac{a_{2}}{v}$. If the same procedure as previous is taken, a new predefined distance should be

$$
R_{\text {safe }}=R+v \tau_{2}
$$

Therefore, in order to make our protocol safe against asynchronous maneuvers both at a resolution starting point and at a new waypoint, from equation (23) and (24), a new predefined safety distance should be

$$
R_{\text {safe }}=R+v\left(\tau_{1}+\tau_{2}\right)
$$


Then, a new protocol for exact and inexact conflicts can be obtained from equation (9) with the new safety distance in equation (25). For example, if we consider a B747 cruising with $\mathrm{M}=8$ at $40,000 \mathrm{ft}$ altitude and the maximum allowable time discrepancy both at a resolution starting point and at a new waypoint is $\tau_{1}=10 \mathrm{sec}$ and $\tau_{2}=10 \mathrm{sec}$ respectively, then a new safety distance is $R_{\text {safe }}=7.53 \mathrm{~nm}$.

\section{Conclusions}

Protocol-based $N$-aircraft conflict resolution for air traffic control has been proposed in this paper. This work is motivated by the free flight concept which allows aircraft to fly user-preferred trajectories; in such a situation, automatic conflict detection and resolution is crucial. We assume that aircraft have nominal trajectories which are at constant altitude and heading, with possibly varying velocities, and we assume that all resolution takes place in the horizontal plane. All aircraft are assumed to share information about their position, velocity, and heading.

We define an exact conflict to be the case in which all aircraft trajectories collide at a point in space and time. While the assumption of an exact conflict is unrealistic for more than two aircraft, its solution provides valuable insight and motivation into the solution for the general case. Considering resolution maneuvers which are triangular deviations from the nominal trajectories parametrically defined by the magnitude of the heading change, we compute an analytic solution to the minimum heading change for all aircraft involved in an exact conflict, to safely resolve the conflict.

We define an inexact conflict to be one in which the aircraft trajectories collide at multiple points in space and time, and we consider two classes of inexact conflict. The first class is a perturbation of the exact conflict, in which all conflict points lie within a circle whose diameter is equal to the required separation distance between aircraft: our solution to this case is a perturbation of the solution to the exact conflict. In the general inexact conflict, we generalize our resolution maneuver and consider heading and/or velocity change to resolve the conflict. 
We analyze the robustness of the protocols with respect to uncertainties in aircraft's position, velocity, and heading. For example, we show that the protocols are still safe even when a sharp corner is replaced by a circular arc corresponding to a constant bank angle turn. Finally, we validate our resolutions through simulation using a dynamic aircraft model.

The benefits of this protocol-based method over previously designed methods are that it applies to $N$ aircraft conflicts with very few restrictions, that as it is based on an analytic solution, it requires little numerical computation and thus can be used in real-time, and that it is simple to understand (for pilots) and to design embedded software implementations (for autopilots). Even though we propose the protocol-based conflict resolution methodology for airborne conflict avoidance under the free flight environment, our protocol could be implemented for an immediate use in today's air traffic control systems. For example, it could be used as a backup conflict resolution algorithm for current air traffic control systems.

Finally, the protocol may be simply extended to three dimensional airspace by projecting the nominal trajectories onto the horizontal plane, applying our protocol, and then back projecting with constant flight path angle. Since all conflicts in three dimensional airspace is included in the conflicts in the projected horizontal plane, the projected two dimensional resolution guarantees safety. The result is conservative, yet we are currently studying less conservative implementations of our protocol in three dimensions. In addition, our current research involves analyzing the effects of finite and varying information horizons on our protocol.

\section{Appendix A: Proof of Lemma 1}

Proof: Denote by $S_{1}$ the statement: there are no conflicts over $t \in\left[0, T_{f}\right]$; and by $S_{2}$ the statement $\left(C_{1} \wedge C_{2}\right) \wedge\left(C_{3} \wedge C_{4}\right) \wedge \cdots \wedge\left(C_{N(N-1)-1} \wedge C_{N(N-1)}\right)$.

(1) Show (if) is true first i.e., $S_{2} \Rightarrow S_{1}$.

Assume $S_{2}$ is not true. This means at least, one of $C_{i}(i=1, \cdots, N(N-1))$ is false. Without loss of generality, we suppose $C_{3}$ is false, i.e., $\left(d_{23}^{1}\right)_{\min }(t)<R$. Then, there exists $t \in\left[0, \frac{T_{f}}{2}\right]$ such that $\left(d_{23}^{1}\right)_{\min }(t)=d(t)<R$ at some 
$t \in\left[0, \frac{T_{f}}{2}\right]$. From the definition of a conflict, there is a conflict over $\left[0, \frac{T_{f}}{2}\right]$, thus, over $\left[0, T_{f}\right]$. This is a contradiction to $S_{1}$. Hence, $S_{2} \Rightarrow S_{1}$ is true.

(2) Show (only if) is true, i.e., $S_{1} \Rightarrow S_{2}$.

Assume there is at least one conflict over $\left[0, T_{f}\right]$. Without loss of generality, we suppose a conflict occurs between aircraft 1 and aircraft 3 . This means that $d_{13}(t)<R$ at some $t \in\left[0, T_{f}\right]$. We consider two cases. First, we assume a conflict occurs at some $t \in\left[0, \frac{T_{f}}{2}\right]$. Then, $d_{13}(t)<R$ is true at some $t \in\left[0, \frac{T_{f}}{2}\right]$. Since $\left(d_{13}^{1}\right)_{\min }(t) \leq d_{13}(t)$ at all $t \in\left[0, \frac{T_{f}}{2}\right],\left(d_{13}^{1}\right)_{\min }(t)<R$ is true at all $t \in\left[0, \frac{T_{f}}{2}\right]$. This is a contradiction to $S_{2}$. Hence, $S_{1} \Rightarrow S_{2}$. Second, we suppose a conflict occurs at $t \in\left(\frac{T_{f}}{2}, T_{f}\right]$. Then, $d_{13}(t)<R$ occurs at some $t \in\left(\frac{T_{f}}{2}, T_{f}\right]$. Since $\left(d_{13}^{2}\right)_{\min }(t) \leq d_{13}(t)$ over $\left(\frac{T_{f}}{2}, T_{f}\right],\left(d_{13}^{2}\right)_{\min }(t)<R$ is true at all $t \in\left[0, \frac{T_{f}}{2}\right]$. This is a contradiction to $S_{2}$. Hence, $S_{1} \Rightarrow S_{2}$.

Therefore, $S_{1} \Leftrightarrow S_{2}$.

\section{Appendix B: Validation with dynamic aircraft model}

The protocols for multiple-aircraft conflict resolution have been derived using a kinematic aircraft model. However, validation with a dynamic aircraft model is necessary to show applicability of the protocols to real air traffic control problems. The lateral motion of an aircraft is controlled by the aileron $\delta a(\mathrm{rad})$, and the rudder $\delta r(\mathrm{rad})$. The important variables of an aircraft's lateral motion are sideslip velocity $v$ (ft/sec), yaw rate $r(\mathrm{rad} / \mathrm{sec})$, roll rate $p(\mathrm{rad} / \mathrm{sec})$, roll angle $\phi$ ( $\mathrm{rad})$, and heading angle $\psi(\mathrm{rad})$.

A linear lateral dynamic model of a B747 which cruises with $M=0.8$ 
at 40,000 ft altitude is used for validation[5]:

$$
\begin{aligned}
{\left[\begin{array}{c}
\dot{v} \\
\dot{r} \\
\dot{p} \\
\dot{\phi} \\
\dot{\psi}
\end{array}\right]=\left[\begin{array}{ccccc}
-0.0558 & -7.74 & 0 & 0.322 & 0 \\
0.0773 & -0.115 & -0.0318 & 0 & 0 \\
-0.394 & 0.388 & -0.465 & 0 & 0 \\
0 & 0 & 1 & 0 & 0 \\
0 & 1 & 0 & 0 & 0
\end{array}\right]\left[\begin{array}{l}
v \\
r \\
p \\
\phi \\
\psi
\end{array}\right]+\left[\begin{array}{cc}
0 & 0.0564 \\
0.00775 & -0.475 \\
0.143 & 0.153 \\
0 & 0 \\
0 & 0
\end{array}\right]\left[\begin{array}{c}
\delta a \\
\delta r
\end{array}\right] } \\
y=\left[\begin{array}{lllll}
1 & 0 & 0 & 0 & 0 \\
0 & 0 & 0 & 0 & 1
\end{array}\right]\left[\begin{array}{l}
r \\
p \\
\phi \\
\psi
\end{array}\right]
\end{aligned}
$$

If we define $x:=[v r p \phi \psi]^{T}, \delta:=\left[\delta_{a} \delta_{r}\right]^{T}$ and $y:=[v \psi]$, equation (26) can be written in the standard linear state-space equation such as:

$$
\begin{aligned}
\dot{x} & =A x+B \delta \\
y & =C x
\end{aligned}
$$

where $A, B$ and $C$ correspond to matrices in equation (26) accordingly.

In Figure 11-(a), the trajectory generator produces a conflict resolution trajectory according to the corresponding protocol for multiple-aircraft conflict resolution and the controller keeps the aircraft on the conflict resolution trajectory. The control law is:

$$
\begin{gathered}
\delta=-K x+\left(N_{u}+K N_{x}\right) y_{r} \\
\text { where, }\left[\begin{array}{l}
N_{x} \\
N_{u}
\end{array}\right]=\left[\begin{array}{cc}
A & B \\
C & \mathbf{0}
\end{array}\right]^{-1}\left[\begin{array}{l}
\mathbf{0} \\
\mathbf{1}
\end{array}\right]
\end{gathered}
$$

The feedforward control in equation (28) makes the aircraft follow the conflict resolution trajectory and the feedback control $K$ regulates the error dynamics. The feedback control gain $K$ is designed using linear optimal control (LQR). All validation results in Section 4 and Section 5 have been obtained with the closed-loop dynamic model in Figure 11-(a).

We validate our protocol for randomly generated five-aircraft conflict situations with different parameters and a dynamic aircraft model. For each 


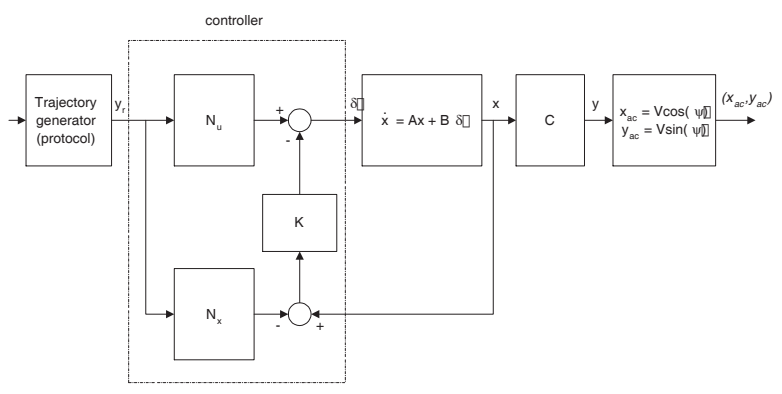

(a) Block diagram of closed-loop lateral dynamics with a LQR controller for B747.
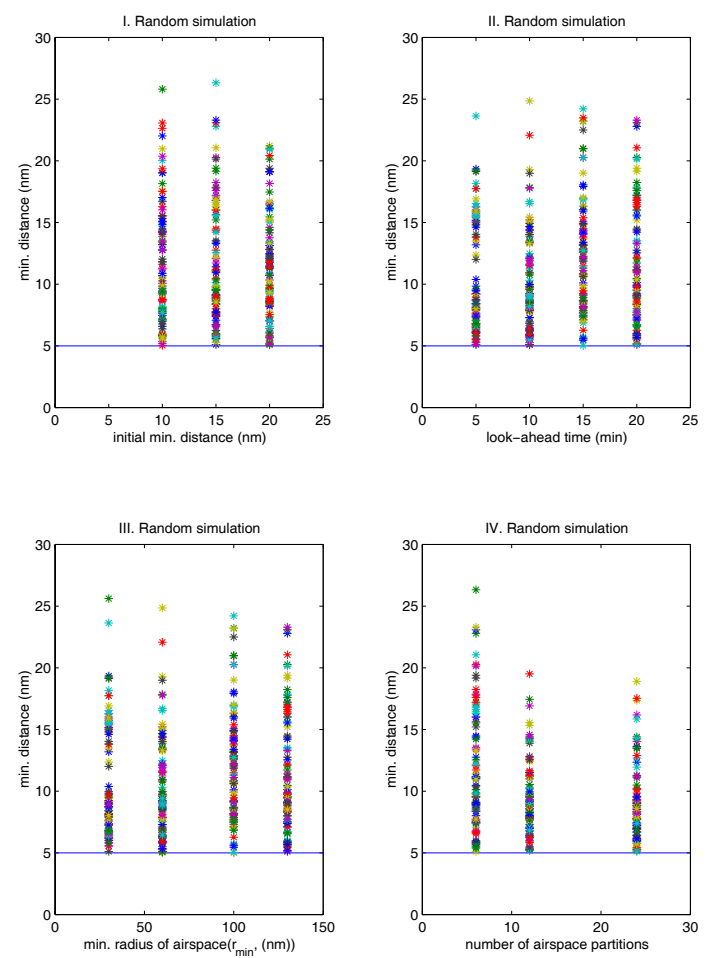

(b) Random simulation with different parameters and a dynamic aircraft model.

Figure 11: Validation with a dynamic aircraft model. 
parameter, simulation has been done for 200 different conflict configurations. Figure 11-(b:(I)) shows random simulation results with different initial minimum distance such as $2 R, 3 R$, and $4 R$, and Figure 11-(b:(II)) shows random simulation results with different look-ahead time such as 5min, 10min, 15min, and 20min. Figure 11-(b:(III)) shows random simulation results with different minimum radius of airspace partition $\left(r_{\text {min }}\right)$ such as $30 \mathrm{~nm}$, $60 \mathrm{~nm}, 100 \mathrm{~nm}$, and 130 $\mathrm{nm}$ and Figure 11-(b:(IV)) shows random simulation results with different number of airspace partitions such as 6, 12, and 24 partitions. All random simulation results show that our protocol resolves all conflicts safely.

\section{References}

[1] J.M. Alliot, N. Durand, and G Granger. Faces: a free flight autonomous and coordinated embarked solver. In Proceedings of the 2nd USA/EUROPE Air Traffic Management RED Seminar, December 1998 .

[2] A. Bicchi and L. Pallottino. On optimal cooperative conflict resolution for air traffic management systems. IEEE Transactions on Intelligent Transportation Systems, 1(4):221-232, 2000.

[3] K. Bilimoria, B. Sridhar, and G. Chatterji. Effects of conflict resolution maneuvers and traffic density of free flight. In Proceedings of the AIAA Guidance, Navigation, and Control Conference, San Diego, 1996.

[4] K.D. Bilimoria. A geometric optimization approach to aircraft conflict resolution. In Proceedings of the AIAA Guidance, Navigation, and Control Conference, Denver, August 2000.

[5] A.E. Bryson. Control of Spacecraft and Aircraft. Princeton University Press, 1994.

[6] I. Burdun and O. Parfentyev. Ai knowledge model for self-organizing conflict prevention/resolution in close free-flight air space. In Proceed- 
ings of the IEEE Aerospace Conference, pages 409-428, Snowmass, CO, March 1999.

[7] B. Carpenter and J. Kuchar. Probability-based collision alerting logic for closely-spaced parallel approach. In Proceedings of the AIAA Aerospace Sciences Meeting and Exhibit, Reno, January 1997.

[8] V. Duong, E. Hoffman, L. Floc'hic, and J.P. Nicolaon. Extended flight rules to apply to the resolution of encounters in autonomous airborne separation. Technical report, EUROCONTROL Experimental Center, September 1996.

[9] V. Duong and K. Zeghal. Conflict resolution advisory for autonomous airborne separation in low-density airspace. In Proceedings of the IEEE Conference on Decision and Control, San Diego, December 1997.

[10] N. Durand, J. Alliot, and O. Chansou. An optimizing conflict solver for atc. Air Traffic Quarterly, 3(3):139-161, 1995.

[11] M. Eby and W. Kelly. Free flight separation assurance using distributed algorithms. In Proceedings of the IEEE Aerospace Conference, pages 429-441, Snowmass, CO, March 1999.

[12] P. Faure and V. Duong. On the applicability of free-flight mode in european airspace. In Proceedings of the 2nd USA/EUROPE Air Traffic Management R\&BD Seminar, December 1998.

[13] E. Frazzoli, Z.H. Mao, J.H. Oh, and E. Feron. Resolution of conflicts involving many aircraft via semidefinite programming. Journal of Guidance, Control, and Dynamics, 24(1):79-86, February 2001.

[14] R. Gazit. Aircraft Surveillance and Collision Avoidance Using GPS. PhD thesis, Stanford University, Stanford, CA, August 1996.

[15] C. Goodchild, M.A. Vilaplana, and S. Elefante. Co-operative optimal airborne separation assurance in free flight airspace. In Proceedings of the 3rd USA/EUROPE Air Traffic Management RED Seminar, June 2000 . 
[16] K. Harper, S. Mulgund, S. Guarino, A. Mehta, and G. Zacharias. Air traffic controller agent model for free flight. In Proceedings of the AIAA Guidance, Navigation, and Control Conference, pages 288-301, Portland, OR, August 9-11, 1999.

[17] J. Hoekstra, R. Ruigrok, R. van Gent, J. Visser, B. Gijsbers, M. Valenti, W. Heesbeen, B. Hilburn, J. Groeneweg, and F. Bussink. Overview of nlr free flight project 1997-1999. Technical Report NLR-CR-2000-227, National Aerospace Laboratory (NLR), May 2000.

[18] J. Hoekstra, R. van Gent, and R. Ruigrok. Conceptual design of free flight with airborne separation assurance. In Proceedings of the AIAA Guidance, Navigation, and Control Conference, pages 807-817, Boston, MA, August 1998.

[19] J. How. Lecture notes for robust control analysis and synthesis. Stanford University, 1997-98, 1997/98.

[20] J. Hu, M. Prandini, and S. Sastry. Optimal maneuver for multiple aircraft conflict resolution: a braid point of view. In Proceedings of the IEEE Conference on Decision and Control, pages 4164-4169, Sydney, December 2000.

[21] R. Irvine. Gears conflict resolution algorithm. In Proceedings of the AIAA Guidance, Navigation, and Control Conference, pages 786-796, Boston, MA, August 10-12, 1998.

[22] S. Kahne and I. Frolow. Air traffic management: Evolution with technology. IEEE Control Systems Magazine, 16(4):12-21, 1996.

[23] J. Kosecka, C. Tomlin, G. Pappas, and S. Sastry. Generation of conflict resolution maneuver for air traffic management. In Proceedings of the International Conference on Robotics and Intelligent Systems, Grenoble, September 1997.

[24] J. Krozel and M. Peters. Strategic conflict detection and resolution for free flight. In Proceedings of the IEEE Conference on Decision and Control, pages 1822-1828, San Diego, December 1997. 
[25] J. Krozel, M. Peters, and G. Hunter. Conflict detection and resolution for future air transportation management. CR-97-205944, NASA Tech. Rep., April 1, 1997.

[26] J.K. Kuchar and L.C. Yang. A review of conflict detection and resolution modeling methods. IEEE Transactions on Intelligent Transportation Systems, 1(4), 2000.

[27] R. Lachner. Collision avoidance as a differential game: Real-time approximation of optimal strategies using higher derivatives of the value function. In Proceedings of the IEEE International Conference on Systems, Man, and Cybernetics, pages 2308-2313, Orlando, October 12-15, 1997.

[28] Z. Mao, E. Feron, and K. Bilimoria. Stability of intersecting aircraft flows under decentralized conflict avoidance rules. In Proceedings of the AIAA Guidance, Navigation, and Control Conference, Denver, August 2000.

[29] P.K. Menon and G.D. Sweriduk. Optimal strategies for free-flight air traffic resolution. Journal of Guidance, Control, and Dynamics, 22(2), March-April 1999.

[30] M.S. Nolan. Fundamentals of Air Traffic Control. Brooks/Cole, Wadsworth, 3rd edition, 1998.

[31] R.A. Paielli and H. Erzberger. Conflict probability estimation for free flight. Journal of Guidance, Control, and Dynamics, 20(3), May-June 1997.

[32] T.S. Perry. In search of the future of air traffic control. IEEE Spectrum, 34(8):18-35, 1997.

[33] M Prandini, J. Lygeros, A. Nilim, and S. Sastry. A probabilistic framework for aircraft conflict detection. In Proceedings of the AIAA Guidance, Navigation, and Control Conference, Portland, August 1999. 
[34] R. Schild. Rule Optimization for Airborne Aircraft Separation. PhD thesis, T.U, Wien, 1998.

[35] RTCA Special Committee. Minimum aviation system performance standards for automatic dependent surveillance broadcast (ADS-B). Technical report, January 1998.

[36] C. Tomlin. Hybrid control of Air Traffic Management Systems. PhD thesis, University of California, Berkeley, 1998.

[37] C. Tomlin, G.J. Pappas, and S. Sastry. Conflict resolution for air traffic management: A study in multiagent hybrid systems. IEEE Transactions on Automatic Control, 43(4), 1998.

[38] L.C. Yang and J.K. Kuchar. Prototype conflict alerting system for free flight. Journal of Guidance, Control, and Dynamics, 20(4), July-August 1997.

[39] K. Zeghal. A review of different approaches based on force fields for airborne conflict resolution. In Proceedings of the AIAA Guidance, Navigation, and Control Conference, pages 818-827, Boston, August 1998.

[40] K. Zeghal and J. Ferber. A reactive approach for distributed air traffic control. In Proceedings of the International Conference on Artificial Intelligence and Expert Systems, pages 381-390, Avignon, France, 1993. 